\title{
Observation of New Cycloisomerization Pattern of 1,5-Bisallenes. Catalyst and
}

\section{Substituent Effects}

\author{
Ping Lu and Shengming Ma*
}

State Key Laboratory of Organometallic Chemistry, Shanghai Institute of Organic

Chemistry, Chinese Academy of Sciences, 354 Fenglin Lu, Shanghai 200032, P. R.

China

masm@mail.sioc.ac.cn

\section{Index}

$\begin{array}{ll}\text { I. General Procedure and analytical data for starting materials } 6 & \text { S2 }\end{array}$

II. General Procedure I and analytical data for compounds $\mathbf{7 a}$ and $\mathbf{7 f - 7 j} \quad$ S11

$\begin{array}{ll}\text { III. General Procedure II and analytical data for compounds } \mathbf{7 b} \text { and } \mathbf{7 e} & \text { S15 }\end{array}$

IV. General Procedure III and analytical data for compounds 7c-7d $\quad$ S16

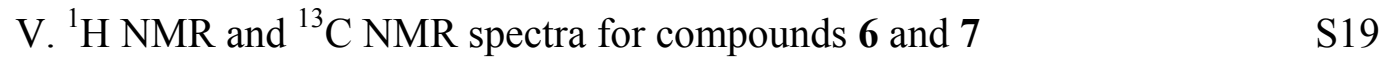

$\begin{array}{ll}\text { VI. NOE spectra for compounds } \mathbf{7 a}, \mathbf{7 h} \text {, and } \mathbf{7 j} & \text { S39 }\end{array}$ 
General Procedure for synthesis of bisallenes $6 a, 6 b$, and $6 e$
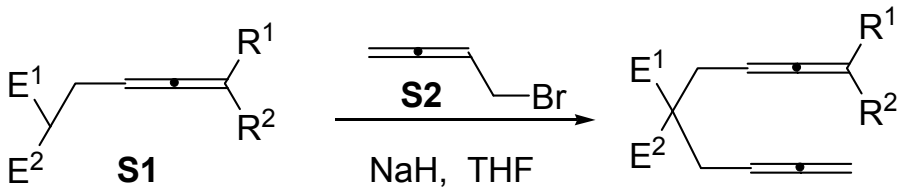

6

S1a, 6a $E^{1}=E^{2}=\mathrm{CO}_{2} \mathrm{Me}, \mathrm{R}^{1}=\mathrm{R}^{2}=\mathrm{Me}$

S1b, 6b $E^{1}=E^{2}=\mathrm{CO}_{2} \mathrm{Me}, \mathrm{R}^{1}, \mathrm{R}^{2}=-\left(\mathrm{CH}_{2}\right)_{5}-$

$\mathrm{S} 1 \mathrm{c}, 6 \mathbf{e} \mathrm{E}^{1}=\mathrm{CO}_{2} \mathrm{Me}, \mathrm{E}^{2}=\mathrm{COMe}, \mathrm{R}^{1}=\mathrm{R}^{2}=\mathrm{Me}$

The bisallenes 6 were prepared from monoallene $\mathbf{S 1}{ }^{1}$ and 4-bromobuta-1,2-diene S2. ${ }^{2}$ To a solution of $\mathbf{S 1}$ in THF was added $\mathrm{NaH}$ (60\% dispersion in mineral oil, 1.5 equiv). After being stirred at $\mathrm{rt}$ for $1 \mathrm{~h}, \mathbf{S 2}$ (1.5 equiv) was added. The solution was stirred at rt overnight. The reaction mixture was quenched with a saturated aqueous $\mathrm{NH}_{4} \mathrm{Cl}$ solution and extracted with diethyl ether three times. The combined organic phase was dried over $\mathrm{MgSO}_{4}$. The solvent was removed by rotary evaporation and the residue was purified by flash chromatography (eluent: petroleum ether/ethyl acetate $=$ 50:1) on silica gel to afford $\mathbf{6 a - 6 \mathbf { b }}$ and $\mathbf{6 e}$.

(1) Dimethyl 2-(buta-2',3'-dienyl)-2-(4'-methylpenta-2',3'-dienyl)malonate (6a)
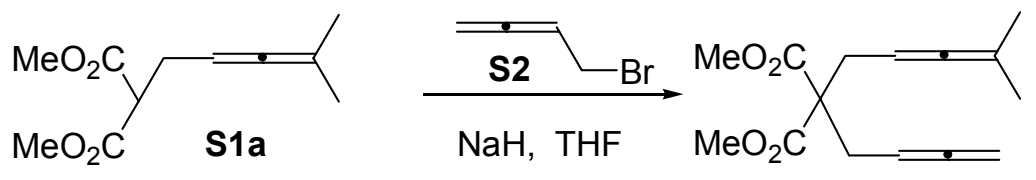

$6 \mathbf{a}$

The reaction of S1a $(0.998 \mathrm{~g}, 4.7 \mathrm{mmol})$ in THF $(20 \mathrm{~mL}), \mathrm{NaH}(60 \%$ dispersion in mineral oil, $291 \mathrm{mg}, 7.1 \mathrm{mmol})$, and $\mathbf{S 2}(942 \mathrm{mg}, 7.1 \mathrm{mmol})$ afforded $1.116 \mathrm{~g} \mathrm{(90 \% )}$ of 6a. ${ }^{1} \mathrm{H}$ NMR (300 MHz, $\left.\mathrm{CDCl}_{3}\right): \delta$ 4.98-4.87 (m, $\left.1 \mathrm{H}\right), 4.79-4.70$ (m, $\left.1 \mathrm{H}\right), 4.64$ (dt, $J=6.6,2.4 \mathrm{~Hz}, 2 \mathrm{H}), 3.71(\mathrm{~s}, 6 \mathrm{H}), 2.65(\mathrm{dt}, J=8.1,2.4 \mathrm{~Hz}, 2 \mathrm{H}), 2.58(\mathrm{~d}, J=7.5 \mathrm{~Hz}$, $2 \mathrm{H}), 1.64(\mathrm{~d}, J=2.7 \mathrm{~Hz}, 6 \mathrm{H}) ;{ }^{13} \mathrm{C} \mathrm{NMR}\left(75.4 \mathrm{MHz}, \mathrm{CDCl}_{3}\right): \delta 20.5,31.5,32.5,52.4$, 
57.9, 74.5, 82.4, 84.1, 95.2, 171.0, 203.7, 209.9; IR (neat) 1956, 1737, 1440, 1284, $1204 \mathrm{~cm}^{-1}$; MS (ESI) $\mathrm{m} / z 265[\mathrm{M}+\mathrm{H}]^{+}$; HRMS calcd. for $\mathrm{C}_{15} \mathrm{H}_{20} \mathrm{O}_{3}$ : 264.1362; found, 264.1357.

(2) Dimethyl 2-(buta-2',3'-dienyl)-2-(4',4'-pentamethylenebuta-2',3'-dienyl)malonate $(\mathbf{6 b})$

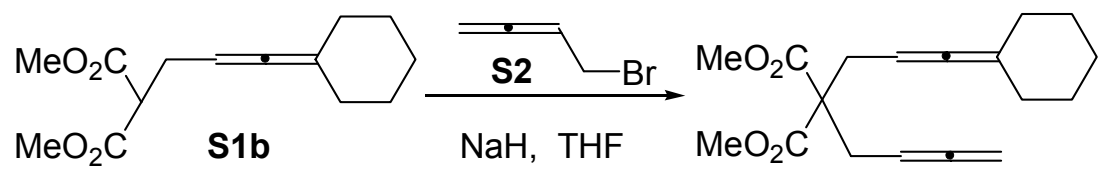

$6 b$

The reaction of $\mathbf{S 1 b}(800 \mathrm{mg}, 3.2 \mathrm{mmol}), \mathrm{NaH}(60 \%$ dispersion in mineral oil, 200 $\mathrm{mg}, 4.8 \mathrm{mmol})$, and $\mathbf{S 2}(612 \mathrm{mg}, 4.8 \mathrm{mmol})$ in $25 \mathrm{~mL}$ of THF afforded $391 \mathrm{mg}$ (41\%) of 6b. ${ }^{1} \mathrm{H}$ NMR (300 MHz, $\left.\mathrm{CDCl}_{3}\right): \delta 5.00-4.89(\mathrm{~m}, 1 \mathrm{H}), 4.80-4.71(\mathrm{~m}, 1 \mathrm{H})$, 4.68-4.62 (m, $2 \mathrm{H}), 3.71(\mathrm{~s}, 6 \mathrm{H}), 2.65(\mathrm{dt}, J=8.1,2.6 \mathrm{~Hz}, 2 \mathrm{H}), 2.60(\mathrm{~d}, J=7.5 \mathrm{~Hz}, 2$ H), 2.10-2.00 (m, $4 \mathrm{H}), 1.65-1.44(\mathrm{~m}, 6 \mathrm{H}) ;{ }^{13} \mathrm{C} \mathrm{NMR}\left(75.4 \mathrm{MHz}, \mathrm{CDCl}_{3}\right): \delta .26 .0$, $27.2,31.4,31.5,32.8,52.4,58.1,74.5,82.3,84.2,102.3,171.0,200.5,210.0$; IR (neat) 1956, 1736, 1440, 1284, $1205 \mathrm{~cm}^{-1}$; MS (ESI) $\mathrm{m} / \mathrm{z} 305[\mathrm{M}+\mathrm{H}]^{+}$; HRMS calcd. for $\mathrm{C}_{18} \mathrm{H}_{24} \mathrm{O}_{4}$ : 304.1675; found, 304.1678.

(3) Methyl 2-acetyl-2-(buta-2',3'-dienyl)-6-methylhepta-4,5-dienoate (6e)
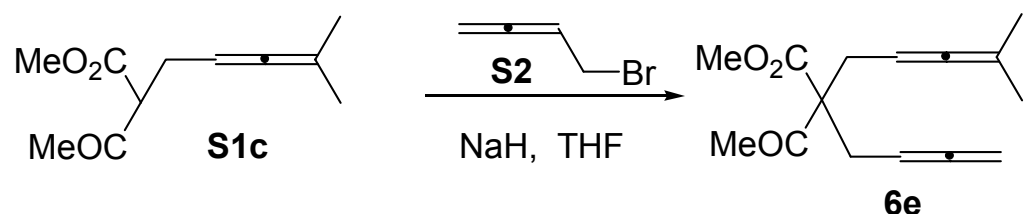

The reaction of S1c (305 mg, $1.0 \mathrm{mmol}), \mathrm{NaH}(60 \%$ dispersion in mineral oil, 150 
mg, $3.2 \mathrm{mmol}$ ), and $\mathbf{S 2}$ (420 mg, $3.1 \mathrm{mmol})$ in $20 \mathrm{~mL}$ of THF afforded $233 \mathrm{mg}$ (60\%) of 6e. ${ }^{1} \mathrm{H}$ NMR $\left(300 \mathrm{MHz}, \mathrm{CDCl}_{3}\right): \delta$ 4.95-4.84 (m, $\left.1 \mathrm{H}\right), 4.77-4.69$ (m, $\left.1 \mathrm{H}\right), 4.65$ (dt, $J=6.6,2.4 \mathrm{~Hz}, 2 \mathrm{H}), 3.72(\mathrm{~s}, 3 \mathrm{H}), 2.72-2.55(\mathrm{~m}, 4 \mathrm{H}), 2.14(\mathrm{~s}, 3 \mathrm{H}), 1.67-1.61(\mathrm{~m}, 6$ $\mathrm{H}) ;{ }^{13} \mathrm{C} \mathrm{NMR}\left(75.4 \mathrm{MHz}, \mathrm{CDCl}_{3}\right) ; \delta 20.4,20.5,26.8,30.6,31.7,52.4,63.8,74.6,82.5$, 84.2, 95.4, 171.8, 203.6, 203.8, 209.8; IR (neat) 1956, 1743, 1715, 1440, $1200 \mathrm{~cm}^{-1}$; MS (ESI) $m / z 249[\mathrm{M}+\mathrm{H}]^{+}, 231\left[\mathrm{M}-\mathrm{H}_{2} \mathrm{O}\right]^{+}$; HRMS calcd. for $\mathrm{C}_{15} \mathrm{H}_{20} \mathrm{O}_{3}$ : 248.1412; found, 248.1418 .

\section{General Procedure for synthesis of bisallenes 6c and 6d}
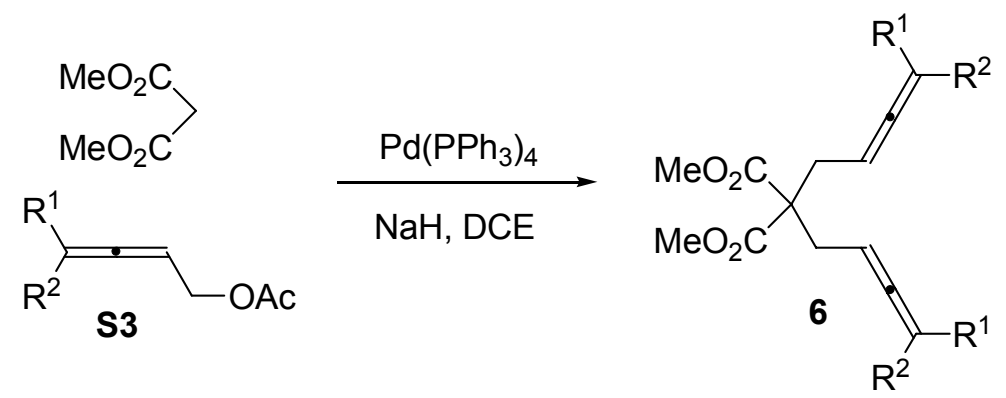

S3a, $6 c \mathrm{R}^{1}=\mathrm{R}^{2}=\mathrm{Me}$

S3b, $6 \mathbf{d ~ R} \mathbf{R}^{1}, \mathrm{R}^{2}=-\left(\mathrm{CH}_{2}\right)_{5}$ -

To a reaction flask were added $\mathrm{Pd}\left(\mathrm{PPh}_{3}\right)_{4}(2.5 \mathrm{~mol} \%), \mathbf{S 3}$ (2.5-3.0 equiv), dimethyl malonate ( 1 equiv), and 1,2-dichloroethane. Then $\mathrm{NaH}(60 \%$ dispersion in mineral oil, 2.5-3.0 equiv) was added under nitrogen. The resulting mixture was stirred at $\mathrm{rt}$ overnight. The solution was quenched with a saturated aqueous $\mathrm{NH}_{4} \mathrm{Cl}$ solution and extracted with $\mathrm{CH}_{2} \mathrm{Cl}_{2}$. The organic layer was dried over $\mathrm{MgSO}_{4}$. The solvent was removed by rotary evaporation and the residue was purified by flash chromatography (eluent: petroleum ether/ethyl acetate $=50: 1$ ) on silica gel to afford $\mathbf{6 c}$ and $\mathbf{6 d}$.

(1) Dimethyl 2,2-bis(4'-methylpenta-2',3'-dienyl)malonate (6c) 

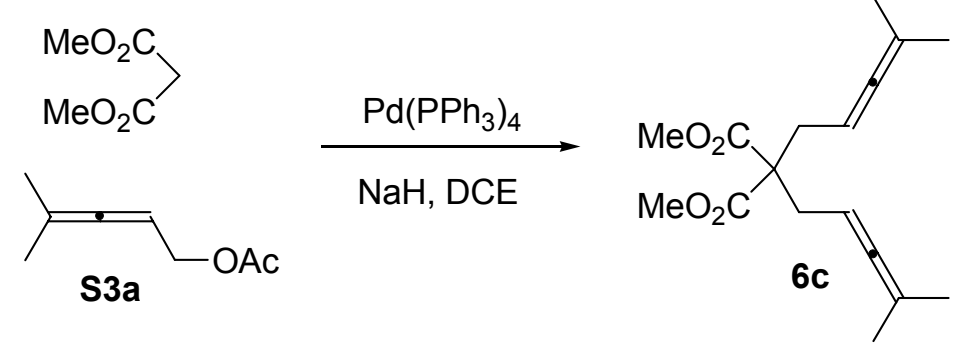

The reaction of $\mathrm{Pd}\left(\mathrm{PPh}_{3}\right)_{4}(35 \mathrm{mg}, 0.025 \mathrm{mmol})$, 4-methylpenta-2,3-dienyl acetate S3a (421 mg, $3.0 \mathrm{mmol})$, dimethyl malonate (132 mg, $1.0 \mathrm{mmol}), 10 \mathrm{~mL}$ of 1,2-dichloroethane, and $\mathrm{NaH}(60 \%$ dispersion in mineral oil, $172 \mathrm{mg}, 3.0 \mathrm{mmol})$ afforded $151 \mathrm{mg}(52 \%)$ 6c. ${ }^{1} \mathrm{H}$ NMR $\left(300 \mathrm{MHz}, \mathrm{CDCl}_{3}\right): \delta 4.81-4.70$ (m, $\left.2 \mathrm{H}\right), 3.71$ (s, $6 \mathrm{H}), 2.60(\mathrm{~d}, J=7.5 \mathrm{~Hz}, 4 \mathrm{H}), 1.64(\mathrm{~d}, J=2.7 \mathrm{~Hz}, 12 \mathrm{H}) ;{ }^{13} \mathrm{C} \mathrm{NMR}(75.4 \mathrm{MHz}$,

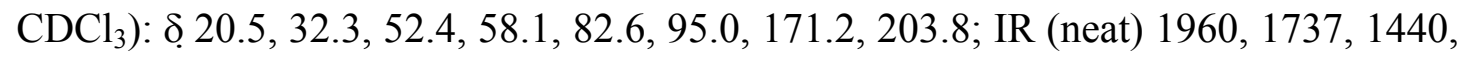
1286, $1203 \mathrm{~cm}^{-1}$; MS (ESI) $\mathrm{m} / z 293[\mathrm{M}+\mathrm{H}]^{+}$; HRMS calcd. for $\mathrm{C}_{17} \mathrm{H}_{24} \mathrm{O}_{4}: 292.1675$; found, 292.1674 .

(2) Dimethyl 2,2-bis(4'4'-pentamethylenebuta-2'3'-dienyl)malonate (6d)
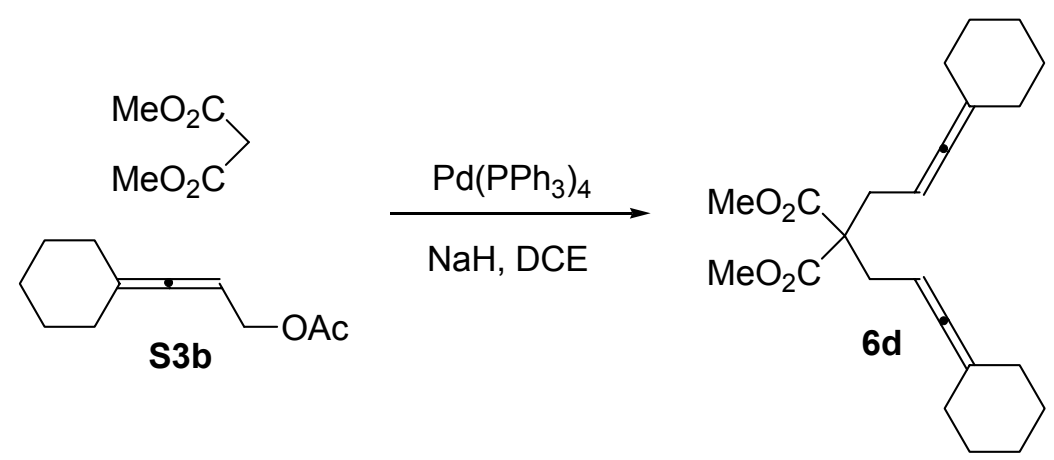

The reaction of $\mathrm{Pd}\left(\mathrm{PPh}_{3}\right)_{4}(67 \mathrm{mg}, 0.05 \mathrm{mmol})$, 3-cyclohexylideneallyl acetate $\mathbf{S 3 b}$ (988 mg, $5.5 \mathrm{mmol})$, dimethyl malonate $(280 \mathrm{mg}, 2.1 \mathrm{mmol}), 40 \mathrm{~mL}$ of 1,2-dichloroethane, and $\mathrm{NaH}(60 \%$ dispersion in mineral oil, $280 \mathrm{mg}, 7.0 \mathrm{mmol})$ afforded $410 \mathrm{mg}$ (52\%) 6d. Solid, m.p. $49-50{ }^{\circ} \mathrm{C}$ (diethyl ether/petroleum ether); ${ }^{1} \mathrm{H}$ NMR (300 MHz, $\left.\mathrm{CDCl}_{3}\right): \delta$ 4.85-4.74 (m, $\left.2 \mathrm{H}\right), 3.70(\mathrm{~s}, 6 \mathrm{H}), 2.62(\mathrm{~d}, J=7.8 \mathrm{~Hz}, 4$ 
$\mathrm{H}), 2.14-2.03(\mathrm{~m}, 8 \mathrm{H}), 1.62-1.46(\mathrm{~m}, 12 \mathrm{H}) ;{ }^{13} \mathrm{C} \mathrm{NMR}\left(75.4 \mathrm{MHz}, \mathrm{CDCl}_{3}\right): \oint$ 26.0, 27.2, 31.4, 32.5, 52.3, 58.2, 82.5, 102.2, 171.2, 200.4; IR (KBr) 1970, 1729, 1445, 1286, $1201 \mathrm{~cm}^{-1}$; MS (ESI) m/z $373[\mathrm{M}+\mathrm{H}]^{+}, 395[\mathrm{M}+\mathrm{Na}]^{+}$; Anal. calcd. for $\mathrm{C}_{23} \mathrm{H}_{32} \mathrm{O}_{4}$ : (\%) C 74.16, H 8.66; found: C 74.51, H 8.79.

\section{General procedure for synthesis of bisallenes $6 f-6 i^{3}$}

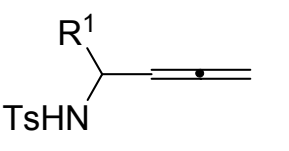

S5a $R^{1}=H$

S5b $R^{1}=M e$

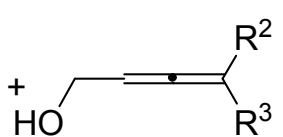

$\mathrm{DEAD}, \mathrm{PPh}_{3}$

S6a $R^{2}=R^{3}=M e$

S6b $R^{2}=R^{3}=E t$

S6c $R^{2}, R^{3}=-\left(\mathrm{CH}_{2}\right)_{5}-$

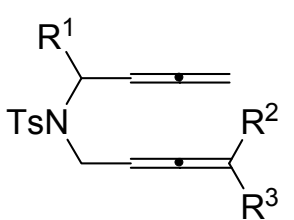

6f $\mathrm{R}^{1}=\mathrm{H}, \mathrm{R}^{2}=\mathrm{R}^{3}=\mathrm{Me}$ $6 g R^{1}=H, R^{2}=R^{3}=E t$ 6h $\mathrm{R}^{1}=\mathrm{H}, \mathrm{R}^{2}, \mathrm{R}^{3}=-\left(\mathrm{CH}_{2}\right)_{5}-$ 6i $\mathrm{R}^{1}=\mathrm{Me}, \mathrm{R}^{2}=\mathrm{R}^{3}=-\left(\mathrm{CH}_{2}\right)_{5}$ -

DEAD (1.5 equiv) was added slowly to a solution of $\mathrm{PPh}_{3}$ (1.5 equiv), S5 (1.0 equiv) and S6 (1.5 equiv) in THF at $\sim-10{ }^{\circ} \mathrm{C}$. The solution was then stirred at $\mathrm{rt}$ and monitored by TLC until S5 was completely consumed. The solvent was removed by rotary evaporation and the residue was purified by flash chromatography (eluent: petroleum ether/ethyl acetate $=50: 1)$ on silica gel to afford $\mathbf{6 f - 6 i}$.

(1) $N$-(Buta-2',3'-dienyl)- $N$-(4'-methylpenta-2',3'-dienyl) 4-tolylsulfonamide (6f)

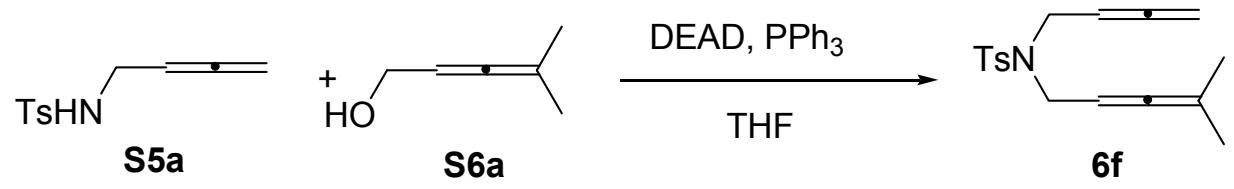

The reaction of $\mathrm{N}$-(buta-2',3'-dienyl) 4-tolylsulfonamide $\mathrm{PPh}_{3}(1.108 \mathrm{~g}, 3.9 \mathrm{mmol})$, S5a (572 mg, 2.6 mmol), 4-methylpenta-2,3-dien-1-ol S6a (377 mg, $3.9 \mathrm{mmol})$, and DEAD (670 mg, $3.9 \mathrm{mmol})$ in THF(10 mL) afforded $563 \mathrm{mg}(72 \%)$ of $6 \mathbf{f}$. Solid, m.p. $35-36{ }^{\circ} \mathrm{C}$ (ethyl acetate/petroleum ether); ${ }^{1} \mathrm{H}$ NMR $\left(300 \mathrm{MHz}, \mathrm{CDCl}_{3}\right): \delta 7.69(\mathrm{~d}, J=$ 
8.7 Hz, 2 H), 7.30-7.25 (m, 2 H), 4.98-4.88 (m, 1 H), 4.80-4.72 (m, 1 H), 4.69 (dt, $J=$ 6.6, 2.4 Hz, $2 \mathrm{H}), 3.90$ (dt, $J=7.2,2.4 \mathrm{~Hz}, 2 \mathrm{H}), 3.81$ (d, $J=7.2 \mathrm{~Hz}, 2 \mathrm{H}), 2.41$ (s, 3 H), $1.63(\mathrm{~d}, J=3.0 \mathrm{~Hz}, 6 \mathrm{H}) ;{ }^{13} \mathrm{C} \mathrm{NMR}\left(75.4 \mathrm{MHz}, \mathrm{CDCl}_{3}\right): \oint$ 20.4, 21.5, 45.1, 46.4, 76.0, 84.1, 85.8, 96.7, 127.1, 129.6, 137.7, 143.1, 203.4, 209.4; IR (neat) 2852, 1955, 1598, 1442, 1344, 1159, $1095 \mathrm{~cm}^{-1}$; MS (ESI) m/z $304[\mathrm{M}+\mathrm{H}]^{+}$; HRMS calcd. for $\mathrm{C}_{17} \mathrm{H}_{21} \mathrm{NO}_{2} \mathrm{~S}: 303.1293$; found, 303.1293.

(2) $N$-(Buta-2',3'-dienyl)-N-(4'-ethylhexa-2',3'-dienyl) 4-tolylsulfonamide (6g)

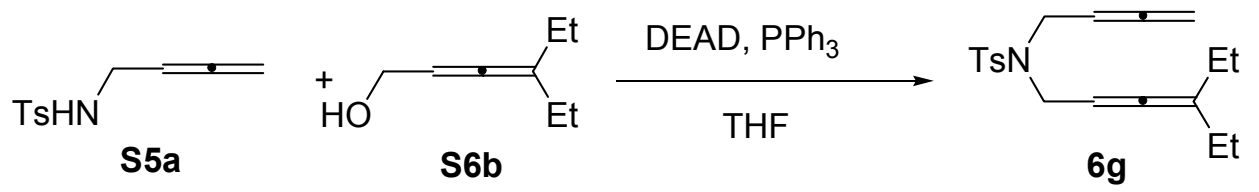

The reaction of $\mathrm{PPh}_{3}$ (530 mg, $\left.2.0 \mathrm{mmol}\right), N$-(buta-2',3'-dienyl) 4-tolylsulfonamide S5a (300 mg, $1.4 \mathrm{mmol})$, 4-ethylhexa-2,3-dien-1-ol S6b (255 mg, $2.0 \mathrm{mmol})$, and DEAD (360 mg, $2.0 \mathrm{mmol})$ afforded $289 \mathrm{mg}(65 \%)$ of $\mathbf{6 g} .{ }^{1} \mathrm{H}$ NMR (300 MHz, $\left.\mathrm{CDCl}_{3}\right): \delta$ 7.74-7.68 (m, $\left.2 \mathrm{H}\right), 7.31-7.25(\mathrm{~m}, 2 \mathrm{H}), 5.04-4.89(\mathrm{~m}, 2 \mathrm{H}), 4.72-4.66(\mathrm{~m}, 2$ H), 3.97-3.90 (m, $2 \mathrm{H}), 3.83$ (d, $J=6.6 \mathrm{~Hz}, 2 \mathrm{H}), 2.41$ (s, $3 \mathrm{H}), 1.93$ (dq, $J=7.5,3.0$ $\mathrm{Hz}, 4 \mathrm{H}), 1.01-0.92(\mathrm{~m}, 6 \mathrm{H}) ;{ }^{13} \mathrm{C} \mathrm{NMR}\left(75.4 \mathrm{MHz}, \mathrm{CDCl}_{3}\right): \delta$ 12.3, 21.5, 25.4, 45.0, $46.7,76.2,85.8,88.4,109.8,127.1,129.6,137.8,143.1,201.7,209.3$; IR (neat) 2867, 1955, 1598, 1453, 1346, 1305, $1160 \mathrm{~cm}^{-1}$; MS (ESI) $m / z 332[\mathrm{M}+\mathrm{H}]^{+}$; HRMS calcd. for $\mathrm{C}_{19} \mathrm{H}_{25} \mathrm{NO}_{2} \mathrm{SNa}^{+}[\mathrm{M}+\mathrm{Na}]^{+}:$354.1507; found, 354.1498.

(3) $N$-(Buta-2',3'-dienyl)-N-(4'4'-pentamethylenebuta-2',3'-dienyl) 4-tolylsulfonamide (6h) 


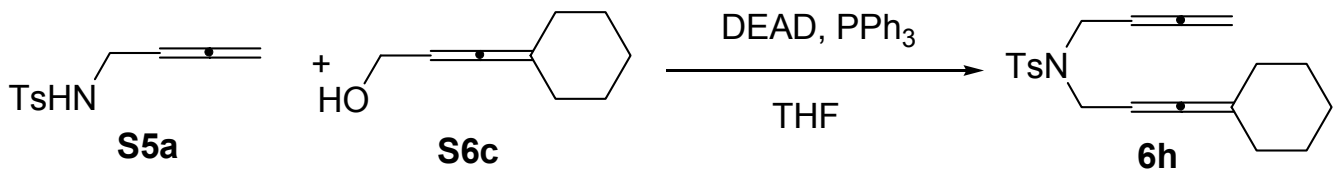

The reaction of $\mathrm{PPh}_{3} \quad(760 \mathrm{mg}, 2.9 \mathrm{mmol}), \quad \mathrm{N}$-(buta-2',3'-dienyl) 4-tolylsulfonamide S5a (426 mg, $1.9 \mathrm{mmol}$ ), 3-cyclohexylideneprop-2-en-1-ol S6c (400 mg, $2.9 \mathrm{mmol}$ ), and DEAD (510 mg, $2.9 \mathrm{mmol})$ afforded $476 \mathrm{mg}(73 \%)$ of $\mathbf{6 h}$. ${ }^{1} \mathrm{H}$ NMR $\left(300 \mathrm{MHz}, \mathrm{CDCl}_{3}\right): \delta 7.70(\mathrm{~d}, J=8.4 \mathrm{~Hz}, 2 \mathrm{H}), 7.28(\mathrm{~d}, J=8.4 \mathrm{~Hz}, 2 \mathrm{H})$, 4.98-4.88 (m, $1 \mathrm{H}), 4.80-4.73(\mathrm{~m}, 1 \mathrm{H}), 4.69$ (dt, $J=6.9,2.4 \mathrm{~Hz}, 2 \mathrm{H}), 3.90(\mathrm{dt}, J=$ 7.2, $2.6 \mathrm{~Hz}, 2 \mathrm{H}), 3.82(\mathrm{~d}, J=7.2 \mathrm{~Hz}, 2 \mathrm{H}), 2.41(\mathrm{~s}, 3 \mathrm{H}), 2.10-2.00(\mathrm{~m}, 4 \mathrm{H})$, 1.65-1.40 (m, $6 \mathrm{H}) ;{ }^{13} \mathrm{C} \mathrm{NMR}\left(75.4 \mathrm{MHz}, \mathrm{CDCl}_{3}\right): \delta$ 21.5, 25.9, 27.1, 31.2, 45.0, 46.6, 76.0, 83.9, 85.7, 103.9, 127.1, 129.6, 137.7, 143.1, 200.1, 209.4; IR (neat) 1956, 1598, 1446, 1346, $1159 \mathrm{~cm}^{-1}$; MS (ESI) $\mathrm{m} / z 344[\mathrm{M}+\mathrm{H}]^{+}$; HRMS calcd. for $\mathrm{C}_{20} \mathrm{H}_{25} \mathrm{NO}_{2} \mathrm{SNa}^{+}$ $[\mathrm{M}+\mathrm{Na}]^{+}:$366.1505; found, 366.1498.

(4) $N$-(4'4'-Pentamethylenebuta-2',3'-dienyl)- $N$-(penta-3',4'-dien-2-yl) 4-tolylsulfonamide $(6 \mathbf{i})$

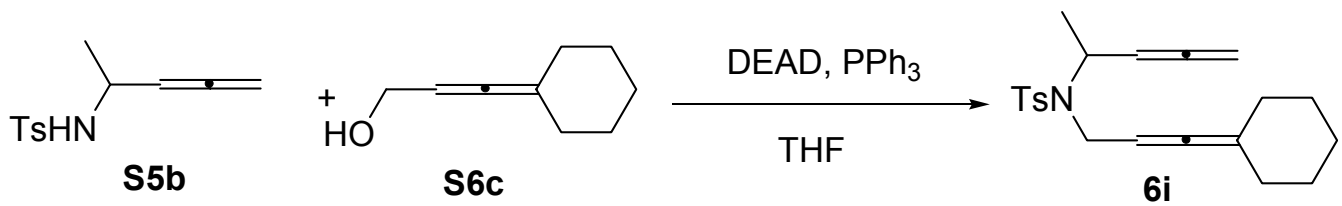

The reaction of $\mathrm{PPh}_{3}(913 \mathrm{mg}, 3.5 \mathrm{mmol}), N$-(penta-3,4-dien-2-yl) 4-tolylsulfonamide S5b (413 mg, 1.7 mmol), 3-cyclohexylideneprop-2-en-1-ol S6c (360 mg, $2.6 \mathrm{mmol})$, and DEAD (609 mg, $3.5 \mathrm{mmol})$ afforded $264 \mathrm{mg}(43 \%)$ of $6 \mathbf{i} .{ }^{1} \mathrm{H}$ NMR (300 MHz, $\left.\mathrm{CDCl}_{3}\right): \delta 7.72(\mathrm{~d}, J=8.4 \mathrm{~Hz}, 2 \mathrm{H}), 7.31-7.25(\mathrm{~m}, 2 \mathrm{H}), 5.10-5.00(\mathrm{~m}, 1$ H), 4.99-4.91 (m, $1 \mathrm{H}), 4.79-4.66(\mathrm{~m}, 2 \mathrm{H}), 4.65-4.55(\mathrm{~m}, 1 \mathrm{H}), 3.82(\mathrm{dd}, J=15.6,6.6$ 
$\mathrm{Hz}, 1 \mathrm{H}), 3.67$ (dd, $J=15.6,6.6 \mathrm{~Hz}, 1 \mathrm{H}), 2.41$ (s, $3 \mathrm{H}), 2.18-2.05$ (m, $4 \mathrm{H}), 1.68-1.45$ (m, $6 \mathrm{H}), 1.22(\mathrm{~d}, J=6.9 \mathrm{~Hz}, 3 \mathrm{H}) ;{ }^{13} \mathrm{C} \mathrm{NMR}\left(75.4 \mathrm{MHz}, \mathrm{CDCl}_{3}\right): \delta$ 18.8, 21.5, 26.0, 27.3, 27.2, 30.8, 31.0, 43.9, 51.7, 77.4, 88.0, 91.9, 104.1, 127.1, 129.6, 138.2, 143.0, 198.6, 208.4; IR (neat) 1955, 1598, 1447, 1346, $1154 \mathrm{~cm}^{-1}$; MS (ESI) $\mathrm{m} / z 358$ $[\mathrm{M}+\mathrm{H}]^{+}$; HRMS calcd. for $\mathrm{C}_{21} \mathrm{H}_{27} \mathrm{NO}_{2} \mathrm{SNa}^{+}[\mathrm{M}+\mathrm{Na}]^{+}$: 380.1674; found, 380.1655 . (S)-N-(4'4'-Pentamethylene-buta-2',3'-dienyl)- $N$-(penta-3,4-dien-2-yl)-4-tolyl sulfonamide ((S)-(-)-6i)

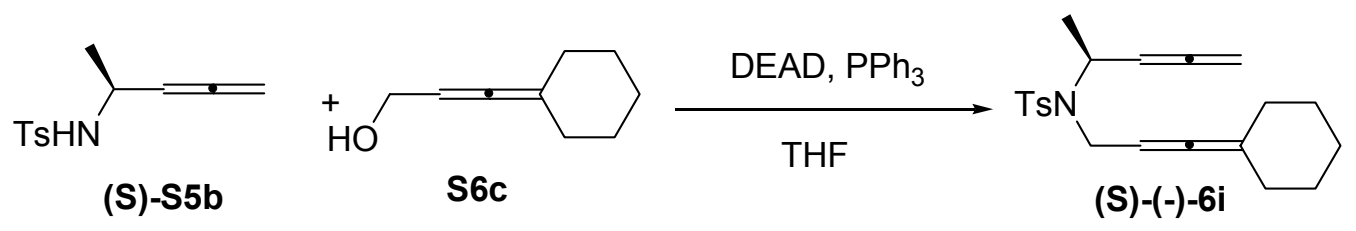

The reaction of (S)-S5b (237 mg, $1 \mathrm{mmol}), \mathrm{PPh}_{3}(393 \mathrm{mg}, 1.5 \mathrm{mmol})$, 3-cyclohexylideneprop-2-en-1-ol S6c (231 mg, $1.67 \mathrm{mmol})$, and DEAD (262 mg, 1.5 mmol) afforded $167 \mathrm{mg}(47 \%, 84 \%$ ee) of (S)-(-)-6i. HPLC conditions: OD-H column; rate, $0.7 \mathrm{~mL} / \mathrm{min}$; eluent, hexane $/ i-\mathrm{PrOH}=100 / 1, \lambda=230 \mathrm{~nm},[\alpha]{ }_{\mathrm{D}}^{20}=-54.5^{\circ}(\mathrm{c}=$ $\left.1.00, \mathrm{CHCl}_{3}\right)$.

\section{Procedure for the synthesis of buta-2',3'-dienyl 4-ethylhexa-2,3-dienyl ether (6j) ${ }^{4}$}

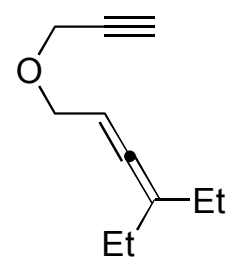

S7

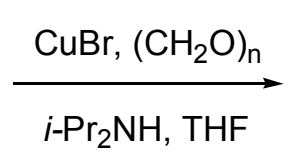

i-Pr $2 \mathrm{NH}, \mathrm{THF}$

A mixture of cuprous bromide (1.140 g, $8.0 \mathrm{mmol})$, paraformaldehyde (964 mg,

$31.8 \mathrm{mmol}$ ), diisopropylamine (1.614 g, $15.9 \mathrm{mmol}), \mathbf{S} 7$ (2.612 g, $15.9 \mathrm{mmol})$, and 20 
$\mathrm{mL}$ of THF were heated to gentle reflux for $5 \mathrm{~h}$. Then the reaction mixture was cooled to rt and filtered through a short column of silica gel. The filtrate was acidified with $6 N$ hydrochloric acid to $\mathrm{pH} 2$ and then extracted with ether $(4 \times 20 \mathrm{~mL})$. The ether extracts are combined, washed with water, and dried over $\mathrm{MgSO}_{4}$. The solvent was removed by rotary evaporation and the residue was purified by flash chromatography (eluent: petroleum ether/ethyl ether $=200: 1-160: 1)$ on silica gel to afford $1.289 \mathrm{~g}$ (46 \%) of 6j. ${ }^{1} \mathrm{H}$ NMR (300 MHz, $\left.\mathrm{CDCl}_{3}\right)$ : $\delta$ 5.29-5.19 (m, $\left.2 \mathrm{H}\right), 4.77$ (dt, $J=6.6,2.4 \mathrm{~Hz}$, $2 \mathrm{H}), 4.03(\mathrm{dt}, J=6.6,2.4 \mathrm{~Hz}, 2 \mathrm{H}), 4.00(\mathrm{~d}, J=6.9 \mathrm{~Hz}, 2 \mathrm{H}), 1.97(\mathrm{dq}, J=7.5,3.0$ $\mathrm{Hz}, 4 \mathrm{H}), 1.00(\mathrm{t}, J=7.5 \mathrm{~Hz}, 6 \mathrm{H}) ;{ }^{13} \mathrm{C} \mathrm{NMR}\left(75.4 \mathrm{MHz}, \mathrm{CDCl}_{3}\right): \delta 12.3,25.4,67.0$, 69.0, 75.5, 87.8, 90.2, 109.0, 201.5, 209.2; IR (neat) 1958, 1457, 1376, $1084 \mathrm{~cm}^{-1}$; MS (ESI) $m / z 179$ [M+H] $]^{+}$HRMS calcd. for $\mathrm{C}_{12} \mathrm{H}_{18} \mathrm{O}$ : 178.1362; found, 178.1358.

\section{References:}

1. Ma, S.; Jiao, N.; Ye, L. Chem. Eur. J. 2003, 9, 6049.

2. 2,3-Butadienyl bromide was prepared from the bromination of 2,3-butadienol. Pornet, J.; Randrianoelina, M. L. J. Organometal. Chem. 1979, 174, 1.

3. Reviews: a) Castro, B. R. Org. React. 1983.29, 1. b) Mitsunobu. O. Synthesis $1981,1$.

4. Crabbé, P.; Nassim, B.; Robert-Lopes, M-T. Org. Synth. 1985, 63, 203; 1990, Coll. Vol. 7, 276. 


\section{General Procedure I (Conditions A):}

A solution of $6(0.25 \mathrm{mmol})$ and $\left[\mathrm{RhCl}(\mathrm{CO})_{2}\right]_{2}(2 \mathrm{~mol} \%)$ in $6 \mathrm{~mL}$ of $\mathrm{CH}_{3} \mathrm{CN}$ was heated under reflux. After the reaction was complete as monitored by TLC (eluent : petroleum ether/ethyl acetate 10:1), the solvent was removed by rotary evaporation and the residue was purified by flash chromatography on silica gel to afford 7 . The following compounds were prepared according to General Procedure I: (1) Dimethyl 5-methylene-4-isopropylidene-2-cycloheptene-1,1-dicarboxylate (7a)

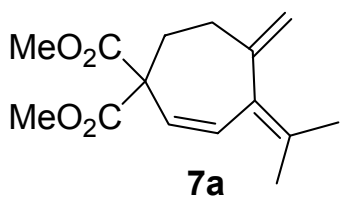

The reaction of $\mathbf{6 a}(66 \mathrm{mg}, 0.25 \mathrm{mmol})$ and $\left[\mathrm{RhCl}(\mathrm{CO})_{2}\right]_{2}(2 \mathrm{mg}, 0.005 \mathrm{mmol})$ in 6 $\mathrm{mL}$ of $\mathrm{CH}_{3} \mathrm{CN}$ afforded $41 \mathrm{mg}(62 \%)$ of $7 \mathbf{a}:{ }^{1} \mathrm{H}$ NMR $\left(300 \mathrm{MHz}, \mathrm{CDCl}_{3}\right): \delta 6.52(\mathrm{~d}$, $J=11.7 \mathrm{~Hz}, 1 \mathrm{H}), 5.60(\mathrm{~d}, J=11.7 \mathrm{~Hz}, 1 \mathrm{H}), 4.89-4.86(\mathrm{~m}, 1 \mathrm{H}), 4.63-4.59(\mathrm{~m}, 1 \mathrm{H})$, $3.71(\mathrm{~s}, 6 \mathrm{H}), 2.49(\mathrm{t}, J=6.6 \mathrm{~Hz}, 2 \mathrm{H}), 2.28(\mathrm{t}, J=6.6 \mathrm{~Hz}, 2 \mathrm{H}), 1.77(\mathrm{~s}, 3 \mathrm{H}), 1.74(\mathrm{~s}$, $3 \mathrm{H}) ;{ }^{13} \mathrm{C}$ NMR $\left(75.4 \mathrm{MHz}, \mathrm{CDCl}_{3}\right): \delta 20.0,21.8,31.1,32.8,52.8,59.6,114.1,124.6$, 131.0, 132.3, 132.6, 147.9, 171.3; IR (neat) 1736, 1633, 1437, 1269, $1228 \mathrm{~cm}^{-1}$; EIMS: $m / z(\%): 264\left(\mathrm{M}^{+}, 11.12\right), 145$ (100); HRMS calcd. for $\mathrm{C}_{15} \mathrm{H}_{20} \mathrm{O}_{4}$ : 264.1362; found, 264.1356.

(2) 5-Isopropylidene-4-methylene-1-tosyl-2,3,4,5-tetrahydro-1 $H$-azepine (7f)

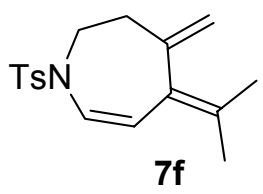

The reaction of $\mathbf{6} \mathbf{f}(77 \mathrm{mg}, 0.25 \mathrm{mmol})$ and $\left[\mathrm{RhCl}(\mathrm{CO})_{2}\right]_{2}(2 \mathrm{mg}, 0.005 \mathrm{mmol})$ in 6 
$\mathrm{mL}$ of $\mathrm{CH}_{3} \mathrm{CN}$ afforded $54 \mathrm{mg}(70 \%)$ of 7f: ${ }^{1} \mathrm{H} \mathrm{NMR}\left(300 \mathrm{MHz}, \mathrm{CDCl}_{3}\right): \delta 7.69(\mathrm{~d}, J$ $=7.5 \mathrm{~Hz}, 2 \mathrm{H}), 7.27(\mathrm{~d}, J=7.5 \mathrm{~Hz}, 2 \mathrm{H}), 6.40(\mathrm{~d}, J=9.2 \mathrm{~Hz}, 1 \mathrm{H}), 5.63(\mathrm{~d}, J=9.2 \mathrm{~Hz}$, $1 \mathrm{H}), 4.64-4.60(\mathrm{~m}, 1 \mathrm{H}), 4.52-4.49(\mathrm{~m}, 1 \mathrm{H}), 3.65(\mathrm{t}, J=6.2 \mathrm{~Hz}, 2 \mathrm{H}), 2.50-2.43(\mathrm{~m}, 2$ H), $2.40(\mathrm{~s}, 3 \mathrm{H}), 1.74(\mathrm{~s}, 3 \mathrm{H}), 1.67(\mathrm{~s}, 3 \mathrm{H}) ;{ }^{13} \mathrm{C} \mathrm{NMR}\left(75.4 \mathrm{MHz}, \mathrm{CDCl}_{3}\right): \delta 20.1$, $21.45,21.54,37.5,48.3,112.3,114.8,125.8,126.8,129.5,130.3,131.0,137.0,143.4$ 146.6; IR (neat) 1673, 1632, 1599, 1347, $1163 \mathrm{~cm}^{-1}$; EIMS: $\mathrm{m} / z(\%): 303\left(\mathrm{M}^{+}, 40.41\right)$, 84 (100); HRMS calcd. for $\mathrm{C}_{17} \mathrm{H}_{21} \mathrm{NO}_{2} \mathrm{~S}$ : 303.1293; found, 303.1289.

(3) 5-(Pent-3-ylidene)-4-methylene-1-tosyl-2,3,4,5-tetrahydro- $1 \mathrm{H}$-azepine (7g)

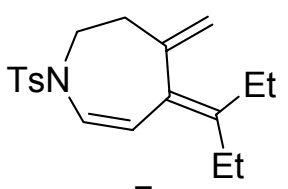

$7 g$

The reaction of $\mathbf{6 g}(83 \mathrm{mg}, 0.25 \mathrm{mmol})$ and $\left[\mathrm{RhCl}(\mathrm{CO})_{2}\right]_{2}(2 \mathrm{mg}, 0.005 \mathrm{mmol})$ in 6 $\mathrm{mL}$ of $\mathrm{CH}_{3} \mathrm{CN}$ afforded $65 \mathrm{mg}\left(78 \%\right.$ ) of $7 \mathbf{g}:{ }^{1} \mathrm{H} \mathrm{NMR}\left(300 \mathrm{MHz}, \mathrm{CDCl}_{3}\right): \oint 7.69$ (d, $J=8.3 \mathrm{~Hz}, 2 \mathrm{H}), 7.27(\mathrm{~d}, J=8.3 \mathrm{~Hz}, 2 \mathrm{H}), 6.40(\mathrm{~d}, J=6.0 \mathrm{~Hz}, 1 \mathrm{H}), 5.64(\mathrm{~d}, J=6.0$ $\mathrm{Hz}, 1 \mathrm{H}), 4.54(\mathrm{~s}, 1 \mathrm{H}), 4.52(\mathrm{~s}, 1 \mathrm{H}), 3.67(\mathrm{t}, J=6.3 \mathrm{~Hz}, 2 \mathrm{H}), 2.47(\mathrm{t}, J=6.3 \mathrm{~Hz}, 2$ H), 2.40 (s, $3 \mathrm{H}), 2.11$ (q, $J=7.5 \mathrm{~Hz}, 2 \mathrm{H}), 2.02$ (q, $J=7.5 \mathrm{~Hz}, 2 \mathrm{H}), 0.96$ (t, $J=7.5$ $\mathrm{Hz}, 3 \mathrm{H}), 0.89$ (t, $J=7.5 \mathrm{~Hz}, 3 \mathrm{H}) ;{ }^{13} \mathrm{C} \mathrm{NMR}\left(75.4 \mathrm{MHz}, \mathrm{CDCl}_{3}\right): \delta$ 13.1, 13.8, 21.4, 23.7, 25.3, 38.1, 48.2, 112.3, 114.1, 126.2, 126.8, 129.5, 130.3, 137.2, 142.0, 143.4, 146.6; IR (neat) 1630, 1598, 1491 1458, 1349, 1184, 1163, $1092 \mathrm{~cm}^{-1}$; EIMS: $\mathrm{m} / z(\%)$ : $331\left(\mathrm{M}^{+}, 42.42\right)$, 91 (100); HRMS calcd. for $\mathrm{C}_{19} \mathrm{H}_{25} \mathrm{NO}_{2} \mathrm{~S}$ : 331.1606; found, 331.1609 . 
(4) 5-Cyclohexylidene-4-methylene-1-tosyl-2,3,4,5-tetrahydro-1H-azepine (7h)

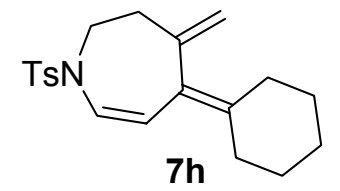

The reaction of $\mathbf{6 h}(86 \mathrm{mg}, 0.25 \mathrm{mmol})$ and $\left[\mathrm{RhCl}(\mathrm{CO})_{2}\right]_{2}(2 \mathrm{mg}, 0.005 \mathrm{mmol})$ in 6 $\mathrm{mL}$ of $\mathrm{CH}_{3} \mathrm{CN}$ afforded $56 \mathrm{mg}(65 \%)$ of $7 \mathbf{h}:{ }^{1} \mathrm{H}$ NMR $\left(300 \mathrm{MHz}, \mathrm{CDCl}_{3}\right): \delta 7.69$ (d, $J=8.7 \mathrm{~Hz}, 2 \mathrm{H}), 7.30-7.24(\mathrm{~m}, 2 \mathrm{H}), 6.40(\mathrm{~d}, J=9.2 \mathrm{~Hz}, 1 \mathrm{H}), 5.68(\mathrm{~d}, J=9.2 \mathrm{~Hz}, 1$ H), $4.57(\mathrm{~d}, J=1.5 \mathrm{~Hz}, 1 \mathrm{H}), 4.49(\mathrm{~d}, J=1.5 \mathrm{~Hz}, 1 \mathrm{H}), 3.68(\mathrm{t}, J=6.3 \mathrm{~Hz}, 2 \mathrm{H}), 2.46$ (t, $J=6.3 \mathrm{~Hz}, 2 \mathrm{H}), 2.41(\mathrm{~s}, 3 \mathrm{H}), 2.25-2.18(\mathrm{~m}, 2 \mathrm{H}), 2.16-2.10(\mathrm{~m}, 2 \mathrm{H}), 1.71-1.38$ $(\mathrm{m}, 6 \mathrm{H}) ;{ }^{13} \mathrm{C} \mathrm{NMR}\left(75.4 \mathrm{MHz}, \mathrm{CDCl}_{3}\right): \delta 21.5,26.7,28.0,28.7,30.3,31.5,38.0$, $48.2,111.7,114.1,126.3,126.8,127.7,129.5,137.1,138.7,143.4,146.4$; IR (neat) 1633, 1599, 1495, 1445, 1398, 1350, $1163 \mathrm{~cm}^{-1}$; EIMS: $\mathrm{m} / z(\%): 343\left(\mathrm{M}^{+}, 11.24\right), 84$ (100); HRMS calcd. for $\mathrm{C}_{20} \mathrm{H}_{25} \mathrm{NO}_{2} \mathrm{~S}$ : 343.1606; found, 343.1607 .

(5) 5-Cyclohexylidene-2-methyl-4-methylene-1-tosyl-2,3,4,5-tetrahydro- $1 H$-azepine (7i)<smiles>C=C1CC(C)N([As])C=CC1=C1CCCCC1</smiles>

The reaction of $6 \mathbf{i}(79 \mathrm{mg}, 0.22 \mathrm{mmol})$ and $\left[\mathrm{RhCl}(\mathrm{CO})_{2}\right]_{2}(2 \mathrm{mg}, 0.005 \mathrm{mmol})$ in 6 $\mathrm{mL}$ of $\mathrm{CH}_{3} \mathrm{CN}$ afforded $46 \mathrm{mg}(58 \%)$ of $7 \mathbf{i}:{ }^{1} \mathrm{H}$ NMR $\left(300 \mathrm{MHz}, \mathrm{CDCl}_{3}\right): \delta 7.69(\mathrm{~d}, J$ $=8.4 \mathrm{~Hz}, 2 \mathrm{H}), 7.25(\mathrm{~d}, J=8.4 \mathrm{~Hz}, 2 \mathrm{H}), 6.22(\mathrm{~d}, J=9.0 \mathrm{~Hz}, 1 \mathrm{H}), 5.90(\mathrm{~d}, J=9.0 \mathrm{~Hz}$, $1 \mathrm{H}), 4.60(\mathrm{~d}, J=0.9 \mathrm{~Hz}, 1 \mathrm{H}), 4.46-4.33(\mathrm{~m}, 2 \mathrm{H}), 2.49(\mathrm{dd}, J=6.5,14.0 \mathrm{~Hz}, 1 \mathrm{H})$, $2.41(\mathrm{~s}, 3 \mathrm{H}), 2.30-2.18$ (m, $4 \mathrm{H}), 2.11(\mathrm{dd}, J=4.4,14.0 \mathrm{~Hz}, 1 \mathrm{H}), 1.61-1.51(\mathrm{~m}, 4 \mathrm{H})$, 
1.50-1.40 (m, $2 \mathrm{H}), 1.17(\mathrm{~d}, J=6.0 \mathrm{~Hz}, 3 \mathrm{H}) ;{ }^{13} \mathrm{C} \mathrm{NMR}\left(75.4 \mathrm{MHz}, \mathrm{CDCl}_{3}\right): \delta$ 19.9, 21.5, 26.7, 28.1, 28.9, 30.6, 31.6, 42.7, 54.6, 115.3, 116.0, 121.6, 127.0, 129.3, 129.6, 137.3, 139.6, 143.0, 143.4; IR (neat) 1632, 1599, 1448, 1406, 1342, $1165 \mathrm{~cm}^{-1}$; EIMS: $m / z(\%): 357\left(\mathrm{M}^{+}, 15.39\right), 43$ (100); HRMS calcd. for $\mathrm{C}_{21} \mathrm{H}_{27} \mathrm{NO}_{2} \mathrm{~S}$ : 357.1763; found, 357.1775;

(6) (S)-5-Cyclohexylidene-2-methyl-4-methylene-1-tosyl-2,3,4,5-tetrahydro- $1 \mathrm{H}$ azepine ((S)-(+)-7i)<smiles>C=C1CC(C)N([Te])C=CC1=C1CCCCC1</smiles>

The reaction of (S)-(-)-6i (89 $\mathrm{mg}, 0.25 \mathrm{mmol}, 84 \mathrm{ee} \%)$ and $\left[\mathrm{RhCl}(\mathrm{CO})_{2}\right]_{2}(2 \mathrm{mg}$, $0.005 \mathrm{mmol})$ in $6 \mathrm{~mL}$ of $\mathrm{CH}_{3} \mathrm{CN}$ afforded $60 \mathrm{mg}(67 \%, 84 \mathrm{ee} \%)$ of $(\mathrm{S})-(+)-7 \mathbf{i}: \mathrm{HPLC}$ conditions: AD-H column; rate, $0.8 \mathrm{~mL} / \mathrm{min}$; eluent, hexane $/ i-\mathrm{PrOH}=100 / 1, \lambda=230$ $\mathrm{nm},[\alpha]^{20}{ }_{\mathrm{D}}=+148.8^{\circ}\left(\mathrm{c}=1.00, \mathrm{CHCl}_{3}\right)$.

(7) 4-Methylene-5-(isopropylidene)-2,3,4,5-tetrahydrooxepine (7j)<smiles>C=C1CCOC=CC1=C(CC)CC</smiles>

The reaction of $\mathbf{6 j}(44 \mathrm{mg}, 0.25 \mathrm{mmol})$ and $\left[\mathrm{RhCl}(\mathrm{CO})_{2}\right]_{2}(2 \mathrm{mg}, 0.005 \mathrm{mmol})$ in 6 $\mathrm{mL}$ of $\mathrm{CH}_{3} \mathrm{CN}$ afforded $27 \mathrm{mg}(61 \%)$ of $7 \mathbf{j}$ (93\% purity by GC). ${ }^{1} \mathrm{H}$ NMR (300 MHz, $\left.\mathrm{CDCl}_{3}\right): \delta 6.19(\mathrm{~d}, J=6.9 \mathrm{~Hz}, 1 \mathrm{H}), 5.32(\mathrm{~d}, J=6.9 \mathrm{~Hz}, 1 \mathrm{H}), 4.98(\mathrm{~d}, J=1.5 \mathrm{~Hz}, 1$ H), $4.77(\mathrm{~d}, J=1.5 \mathrm{~Hz}, 1 \mathrm{H}), 4.12(\mathrm{t}, J=5.7 \mathrm{~Hz}, 2 \mathrm{H}), 2.70(\mathrm{t}, J=5.7 \mathrm{~Hz}, 2 \mathrm{H})$, 
2.23-2.09 (m, $4 \mathrm{H}), 1.01(\mathrm{t}, J=7.5 \mathrm{~Hz}, 3 \mathrm{H}), 0.98(\mathrm{t}, J=7.5 \mathrm{~Hz}, 3 \mathrm{H}) ;{ }^{13} \mathrm{C} \mathrm{NMR}(75.4$ $\left.\mathrm{MHz}, \mathrm{CDCl}_{3}\right): \delta$ 13.0, 14.0, 23.9, 25.3, 39.0, 70.1, 105.7, 112.8, 130.2, 139.6, 146.0, 147.9; IR (neat) 1632, 1601, 1458, 1298, 1270, $1147 \mathrm{~cm}^{-1}$; EIMS: $m / z(\%): 178\left(\mathrm{M}^{+}\right.$, 100); HRMS calcd. for $\mathrm{C}_{12} \mathrm{H}_{18} \mathrm{O}$ : 178.1358; found, 178.1361 .

\section{General Procedure II (Conditions B):}

A solution of $6(0.25 \mathrm{mmol}),[\mathrm{RhCl}(\mathrm{COD})]_{2}(2.5 \mathrm{~mol} \%)$, and dppp $(5 \mathrm{~mol} \%)$ in $\mathrm{CH}_{3} \mathrm{CN}$ was heated under reflux. After the reaction was complete as monitored by TLC (eluent : petroleum ether/ethyl acetate 10:1), the solvent was removed by rotary evaporation and the residue was purified by flash chromatography on silica gel to afford 7.

(1) Dimethyl 4-cyclohexylidene-5-methylene-2-cycloheptene-1,1-dicarboxylate (7b)

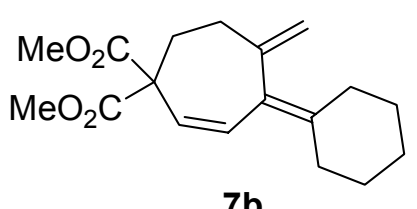

7b

The reaction of $\mathbf{6 b}(76 \mathrm{mg}, 0.25 \mathrm{mmol}),[\mathrm{RhCl}(\mathrm{COD})]_{2}(3 \mathrm{mg}, 0.00625 \mathrm{mmol})$, and dppp (6 mg, $0.0125 \mathrm{mmol})$ in $6 \mathrm{~mL}$ of $\mathrm{CH}_{3} \mathrm{CN}$ afforded $41 \mathrm{mg}(54 \%)$ of $7 \mathbf{b}:{ }^{1} \mathrm{H}$ NMR $\left(300 \mathrm{MHz}, \mathrm{CDCl}_{3}\right): \delta 6.57(\mathrm{~d}, J=11.4 \mathrm{~Hz}, 1 \mathrm{H}), 5.60(\mathrm{~d}, J=11.4 \mathrm{~Hz}, 1 \mathrm{H}), 4.84(\mathrm{~s}, 1$ H), $4.60(\mathrm{~s}, 1 \mathrm{H}), 3.71(\mathrm{~s}, 6 \mathrm{H}), 2.48(\mathrm{t}, J=6.6 \mathrm{~Hz}, 2 \mathrm{H}), 2.29(\mathrm{t}, J=6.6 \mathrm{~Hz}, 2 \mathrm{H})$, 2.28-2.12 (m, $4 \mathrm{H}), 1.60-1.43(\mathrm{~m}, 6 \mathrm{H}) ;{ }^{13} \mathrm{C} \mathrm{NMR}\left(75.4 \mathrm{MHz}, \mathrm{CDCl}_{3}\right): \delta 26.7,28.1$, $28.9,30.3,30.9,31.7,33.2,52.7,59.7,113.3,124.9,129.0,130.5,140.7,147.7,171.2$ IR (neat) 1736, 1633, 1434, $1231 \mathrm{~cm}^{-1}$; EIMS: $m / z(\%): 304\left(\mathrm{M}^{+}, 15.79\right), 91(100)$; HRMS calcd. for $\mathrm{C}_{18} \mathrm{H}_{24} \mathrm{O}_{4}$ : 304.1675 ; found, 304.1675 . 
(2) Methyl 1-acetyl-5-methylene-4-isopropylidene-2-cycloheptene carboxylate (7e)<smiles>C=C1CCC(C(C)=O)(C(C)=O)C=CC1=C(C)C</smiles>

The reaction of $6 \mathrm{e}(62 \mathrm{mg}, 0.25 \mathrm{mmol}),[\mathrm{RhCl}(\mathrm{COD})]_{2}(3 \mathrm{mg}, 0.00625 \mathrm{mmol})$, and dppp (5 mg, $0.0125 \mathrm{mmol})$ in $6 \mathrm{~mL}$ of $\mathrm{CH}_{3} \mathrm{CN}$ afforded $30 \mathrm{mg}(48 \%)$ of $7 \mathrm{e}:{ }^{1} \mathrm{H} \mathrm{NMR}$ $\left(300 \mathrm{MHz}, \mathrm{CDCl}_{3}\right): \oint 6.56(\mathrm{~d}, J=11.4 \mathrm{~Hz}, 1 \mathrm{H}), 5.68(\mathrm{~d}, J=11.4 \mathrm{~Hz}, 1 \mathrm{H}), 4.86(\mathrm{~s}, 1$ H), $4.61(\mathrm{~s}, 1 \mathrm{H}), 3.69(\mathrm{~s}, 3 \mathrm{H}), 2.46(\mathrm{t}, J=6.6 \mathrm{~Hz}, 2 \mathrm{H}), 2.38-2.27(\mathrm{~m}, 1 \mathrm{H}), 2.21(\mathrm{~s}, 3$ H), 2.20-2.08 (m, $1 \mathrm{H}), 1.78(\mathrm{~s}, 3 \mathrm{H}), 1.74(\mathrm{~s}, 3 \mathrm{H}) ;{ }^{13} \mathrm{C} \mathrm{NMR}\left(75.4 \mathrm{MHz}, \mathrm{CDCl}_{3}\right): \delta$ 20.0, 21.7, 27.7, 30.8, 32.8, 52.6, 65.6, 114.7, 125.1, 131.4, 132.6, 132.7, 148.1, 171.8, 205.0; IR (neat) 1746, 1715, 1638, 1434, 1353, 1260, $1224 \mathrm{~cm}^{-1}$; EIMS: $\mathrm{m} / z(\%): 249$ $\left([\mathrm{M}+\mathrm{H}]^{+}, 2.94\right), 206$ (100); HRMS calcd. for $\mathrm{C}_{15} \mathrm{H}_{20} \mathrm{O}_{3}: 248.1412$; found, 248.1408.

\section{General Procedure III (Conditions C):}

A solution of $6(0.25 \mathrm{mmol})$ and $\left[\mathrm{RhCl}(\mathrm{CO})_{2}\right]_{2}(2 \mathrm{~mol} \%)$ in DMF was heated at $110^{\circ} \mathrm{C}$. After the reaction was complete as monitored by TLC (eluent : petroleum ether/ethyl acetate 10:1), then the mixture was diluted with ether, washed with water, and dried over $\mathrm{MgSO}_{4}$ or $\mathrm{Na}_{2} \mathrm{SO}_{4}$. After evaporation, the residue was purified via flash chromatography on silica gel with petroleum ether/ethyl acetate(30:1) as the eluent to afford 7.

(1) Dimethyl 4,5-diisopropylidene-2-cycloheptene-1,1-dicarboxylate (7c) 


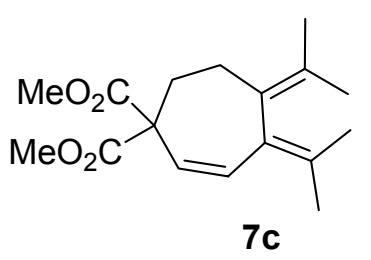

The reaction of of $\mathbf{6 c}(73 \mathrm{mg}, 0.25 \mathrm{mmol})$ and $\left[\mathrm{RhCl}(\mathrm{CO})_{2}\right]_{2}(2 \mathrm{mg}, 0.005 \mathrm{mmol})$ in $6 \mathrm{~mL}$ of DMF was heated at $110^{\circ} \mathrm{C}$ to afford $52 \mathrm{mg}(71 \%)$ of $7 \mathrm{c}:{ }^{1} \mathrm{H}$ NMR $(300 \mathrm{MHz}$, $\left.\mathrm{CDCl}_{3}\right): \oint 6.53(\mathrm{~d}, J=11.3 \mathrm{~Hz}, 1 \mathrm{H}), 5.55(\mathrm{~d}, J=11.3 \mathrm{~Hz}, 1 \mathrm{H}), 3.71(\mathrm{~s}, 3 \mathrm{H}), 3.68(\mathrm{~s}$, $3 \mathrm{H}), 2.72-2.61(\mathrm{~m}, 1 \mathrm{H}), 2.41-2.33(\mathrm{~m} 1 \mathrm{H}), 2.28-2.16$ (m $1 \mathrm{H}), 2.15-2.04(\mathrm{~m}, 1 \mathrm{H})$, $1.74(\mathrm{~s}, 3 \mathrm{H}), 1.59(\mathrm{~d}, J=1.5 \mathrm{~Hz}, 3 \mathrm{H}), 1.56(\mathrm{~s}, 3 \mathrm{H}), 1.44(\mathrm{~d}, J=2.1 \mathrm{~Hz}, 3 \mathrm{H}) ;{ }^{13} \mathrm{C}$ NMR (75.4 MHz, $\left.\mathrm{CDCl}_{3}\right): \delta$ 19.2, 19.8, 20.9, 21.3, 27.3, 31.1, 52.4, 52.9, 60.1, 124.7, 126.6, 131.1, 131.7, 131.87, 131.93, 170.5, 172.3; IR (neat) 1738, 1435, 1371, 1264 , $1224 \mathrm{~cm}^{-1}$; EIMS: $m / z(\%): 292\left(\mathrm{M}^{+}, 42.00\right), 59$ (100); HRMS calcd. for $\mathrm{C}_{17} \mathrm{H}_{24} \mathrm{O}_{4}$ : 292.1675; found, 292.1682.

(2) Dimethyl 4,5-dicyclohexylidene-2-cycloheptene-1,1-dicarboxylate (7d)<smiles>COC(OC)C1(C(=O)O)C=CC(=C2CCCCC2)C(=C2CCCCC2)CC1</smiles>

The reaction of of $\mathbf{6 d}(93 \mathrm{mg}, 0.25 \mathrm{mmol})$ and $\left[\mathrm{RhCl}(\mathrm{CO})_{2}\right]_{2}(2 \mathrm{mg}, 0.005 \mathrm{mmol})$ in $6 \mathrm{~mL}$ of DMF was heated at $110{ }^{\circ} \mathrm{C}$ to afford $52 \mathrm{mg}(56 \%)$ of $7 \mathbf{d}:{ }^{1} \mathrm{H}$ NMR $(300$ $\left.\mathrm{MHz}, \mathrm{CDCl}_{3}\right): \oint 6.55(\mathrm{~d}, J=11.4 \mathrm{~Hz}, 1 \mathrm{H}), 5.54(\mathrm{dd}, J=11.4,1.2 \mathrm{~Hz}, 1 \mathrm{H}), 3.70(\mathrm{~s}, 3$ H), $3.69(\mathrm{~s}, 3 \mathrm{H}), 2.82-2.69(\mathrm{~m}, 1 \mathrm{H}), 2.38-2.22(\mathrm{~m}, 3 \mathrm{H}), 2.22-1.81(\mathrm{~m}, 8 \mathrm{H})$, 1.68-1.28 (m, $12 \mathrm{H}) ;{ }^{13} \mathrm{C} \mathrm{NMR}\left(75.4 \mathrm{MHz}, \mathrm{CDCl}_{3}\right): \delta$ 26.7, 26.85, 26.94, 27.5, 27.6, $27.9,28.1,29.5,30.3,30.8,30.9,31.6,52.3,52.9,60.3,125.2,127.4,128.8,131.8$, 133.6, 139.0, 170.3, 172.2; IR (neat) 1737, 1446, 1273, $1222 \mathrm{~cm}^{-1}$; EIMS: $m / z(\%)$ : 
$372\left(\mathrm{M}^{+}, 37.96\right), 41$ (100); HRMS calcd. for $\mathrm{C}_{23} \mathrm{H}_{32} \mathrm{O}_{4}$ : 372.2301 ; found, 372.2296. 


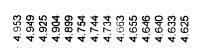

WUVWU

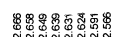

(U)
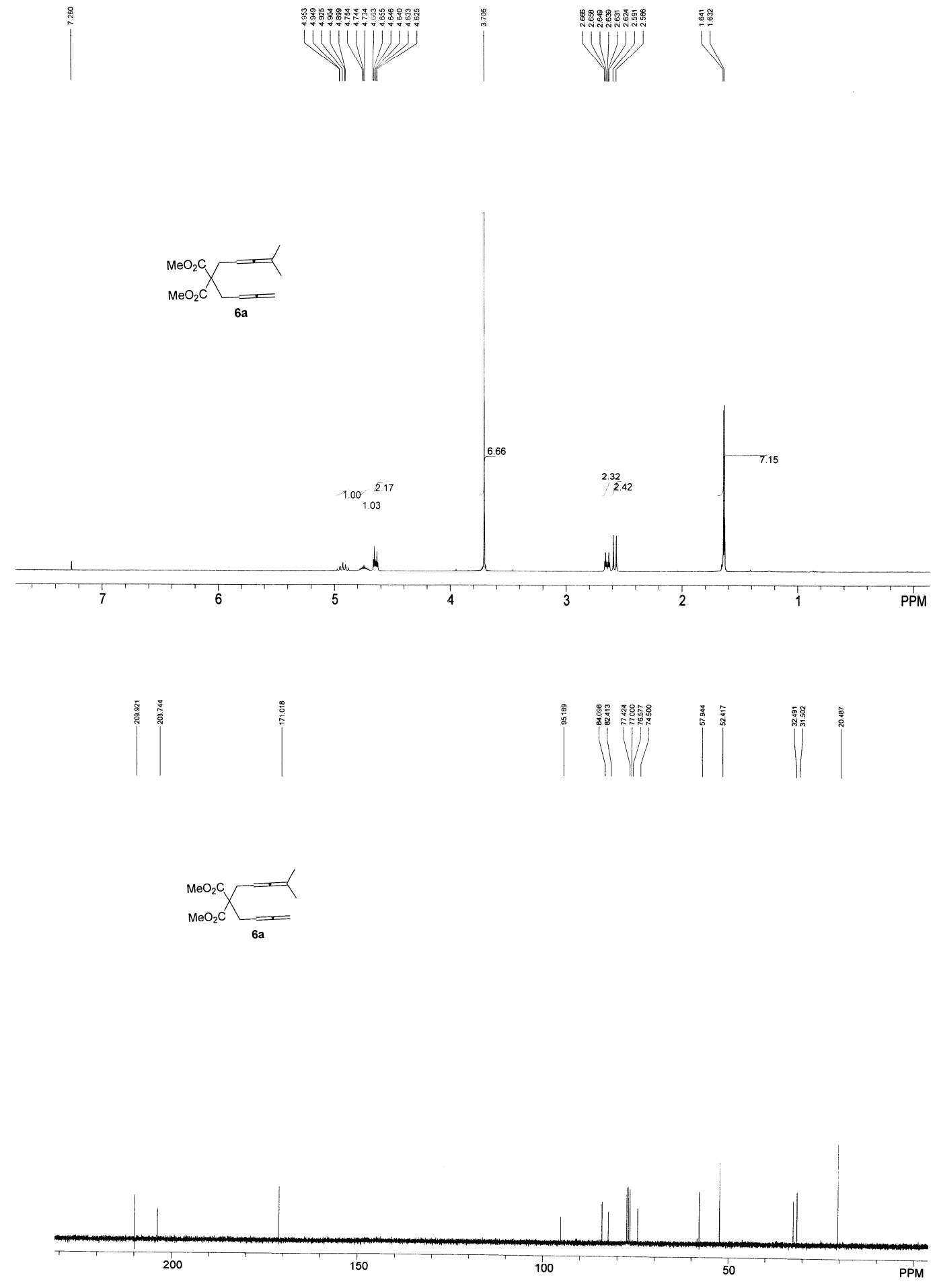

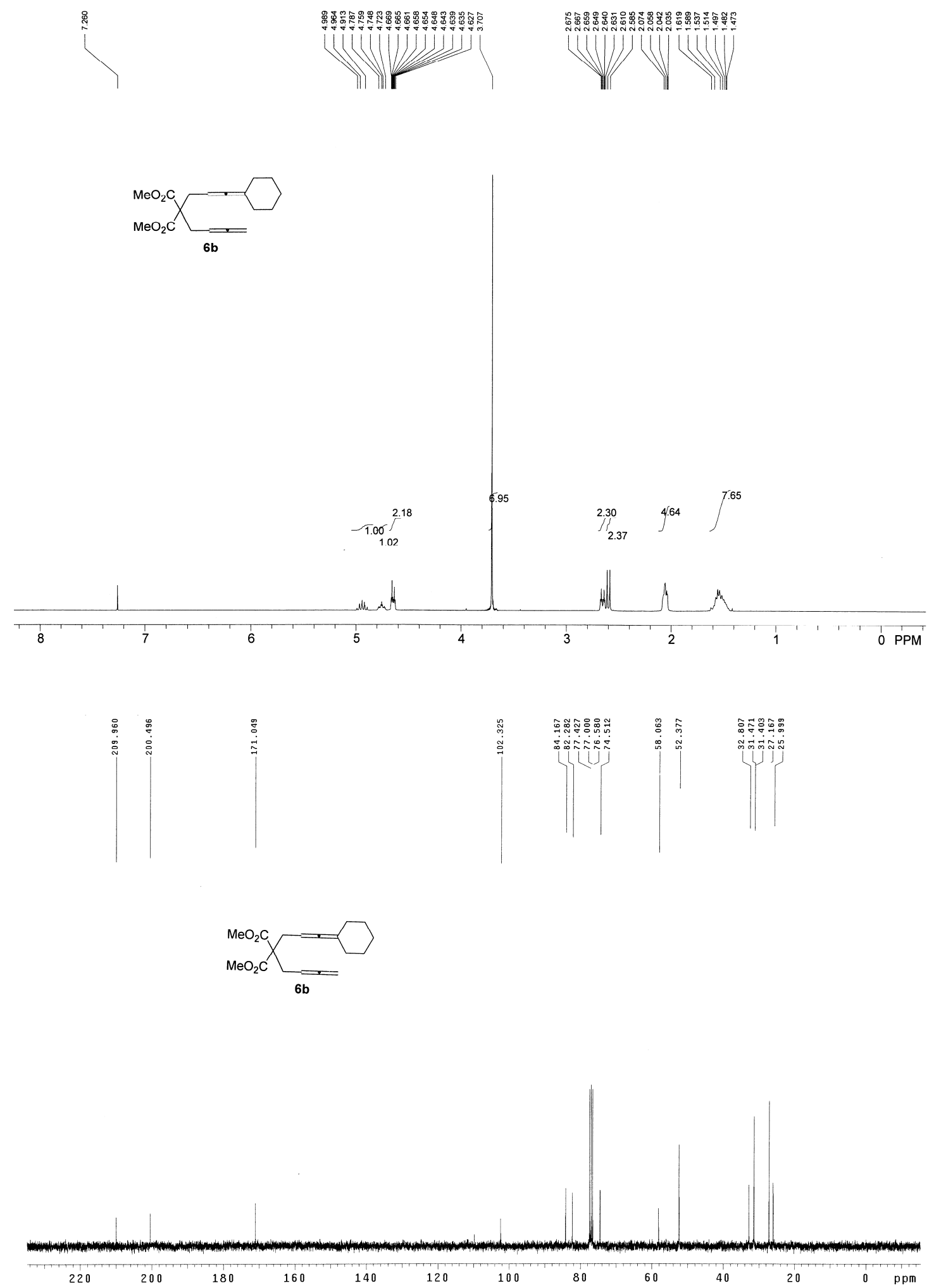

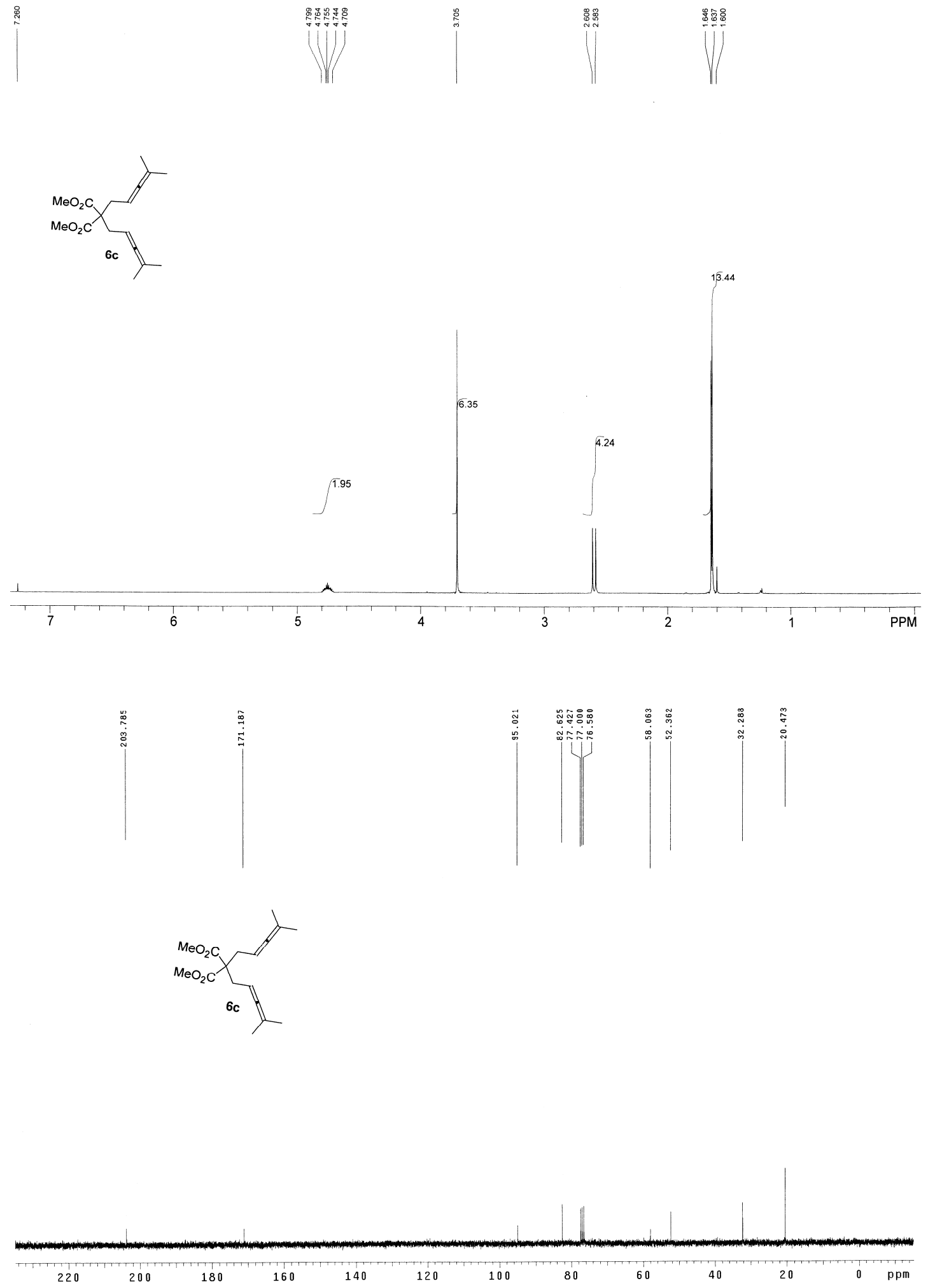

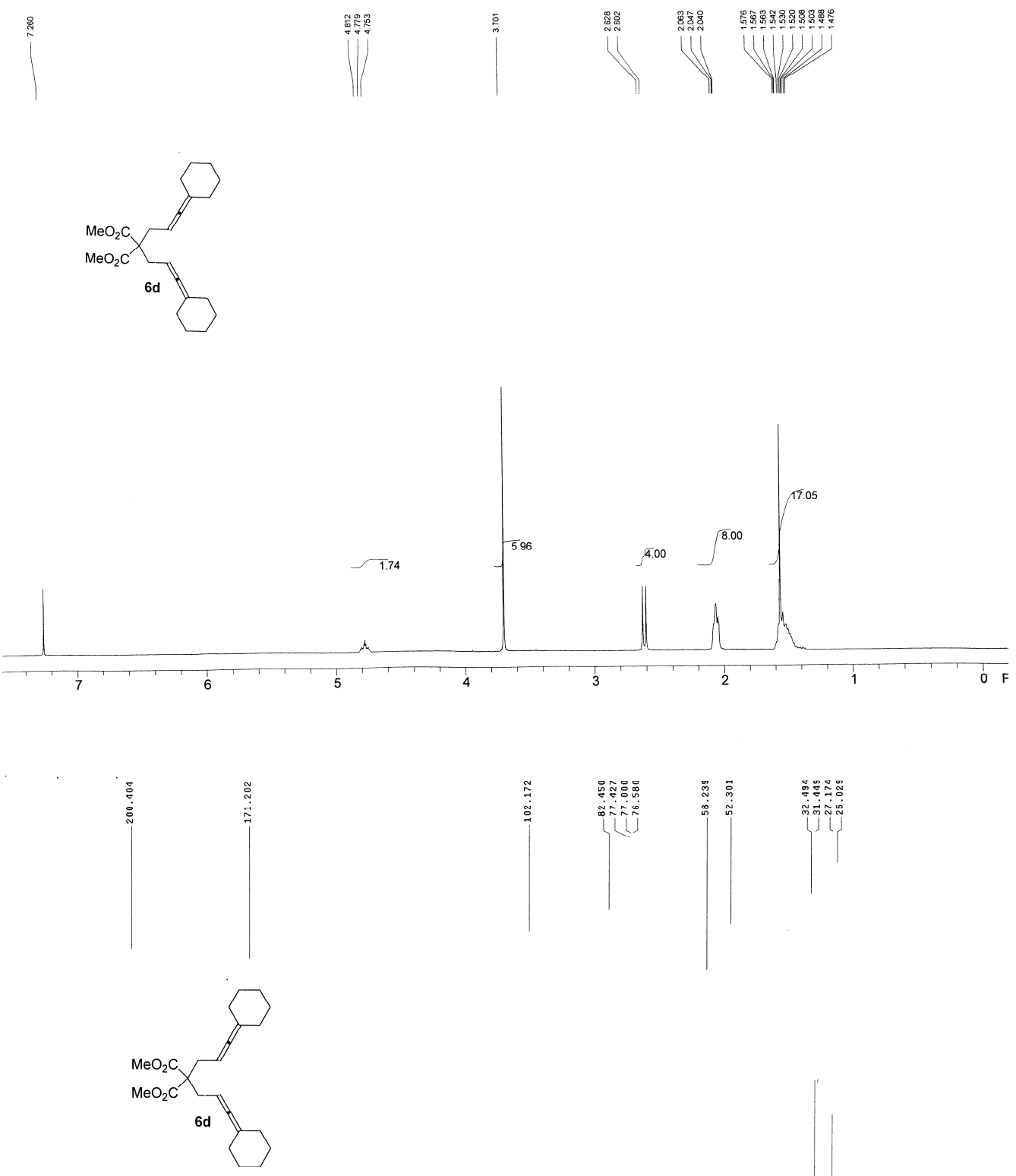

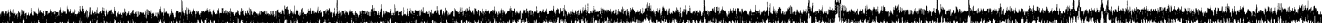
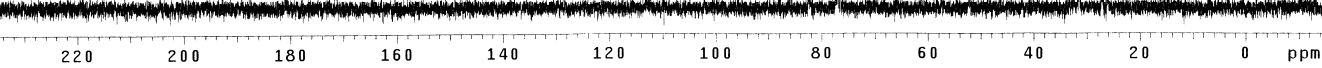

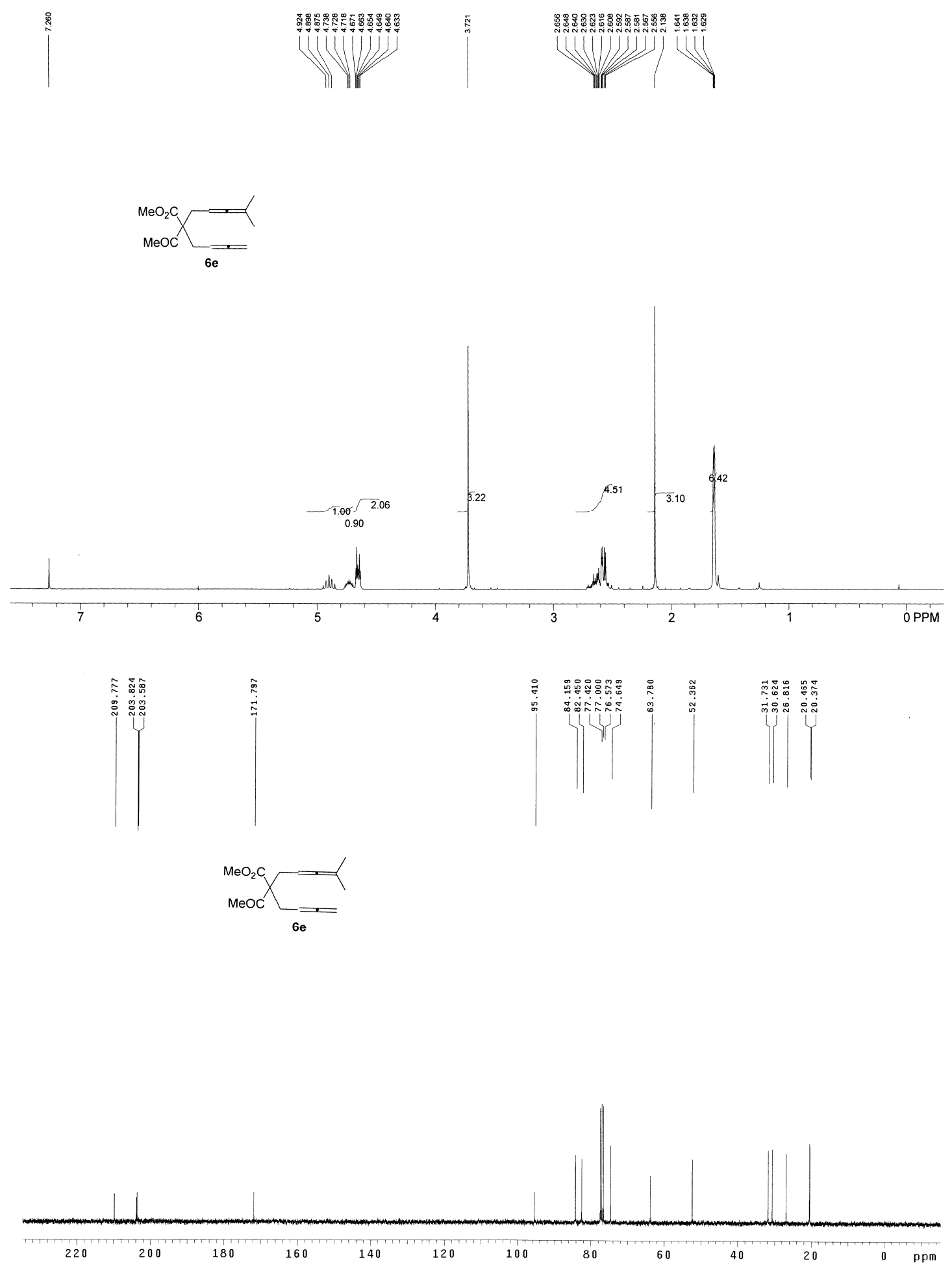


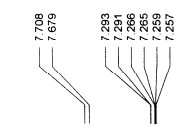

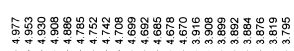

HUW WU

$\left.\right|^{\substack{8 \\ 2}}$

$\sqrt[T s]{{ }_{6 f}}$
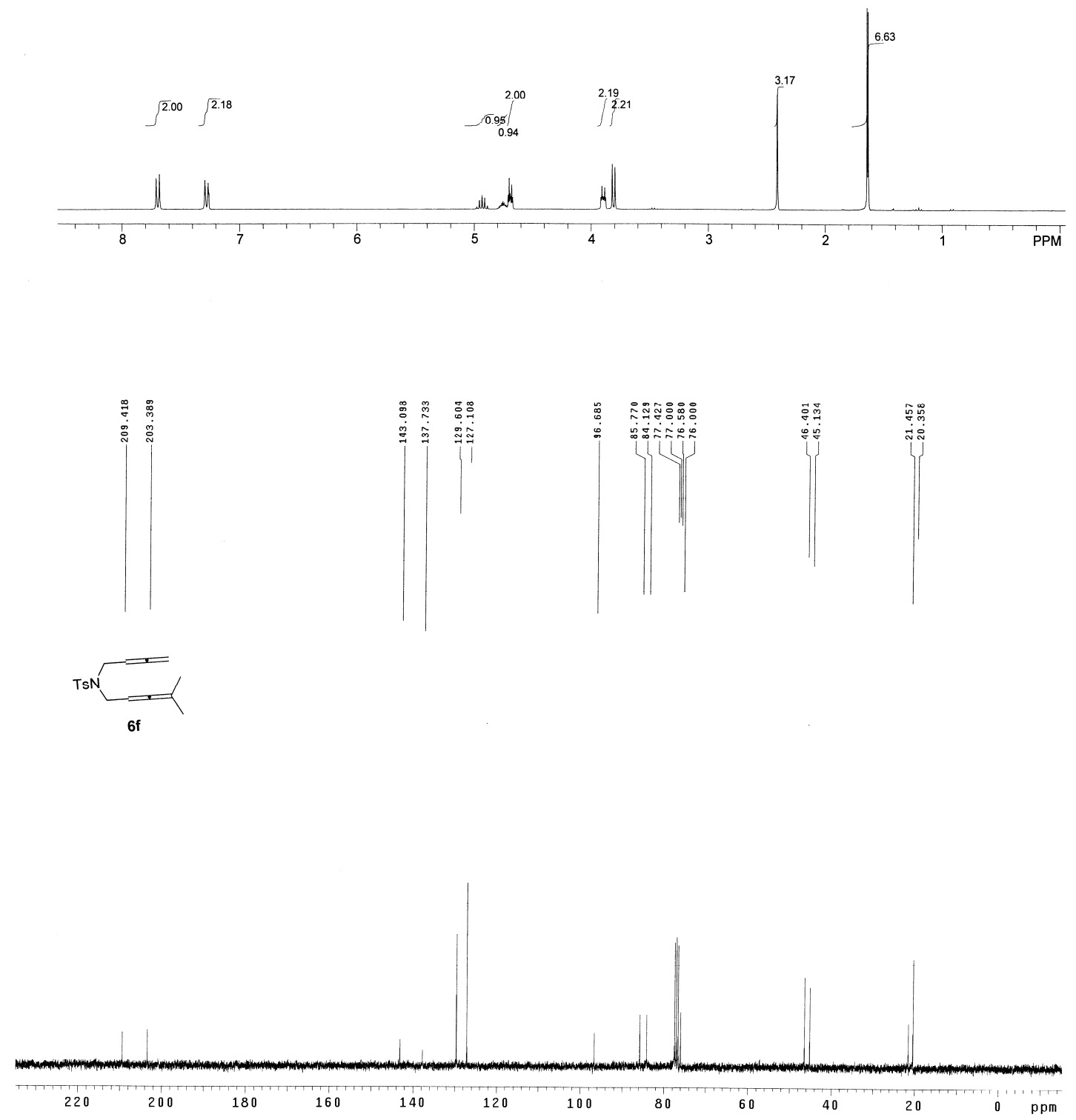

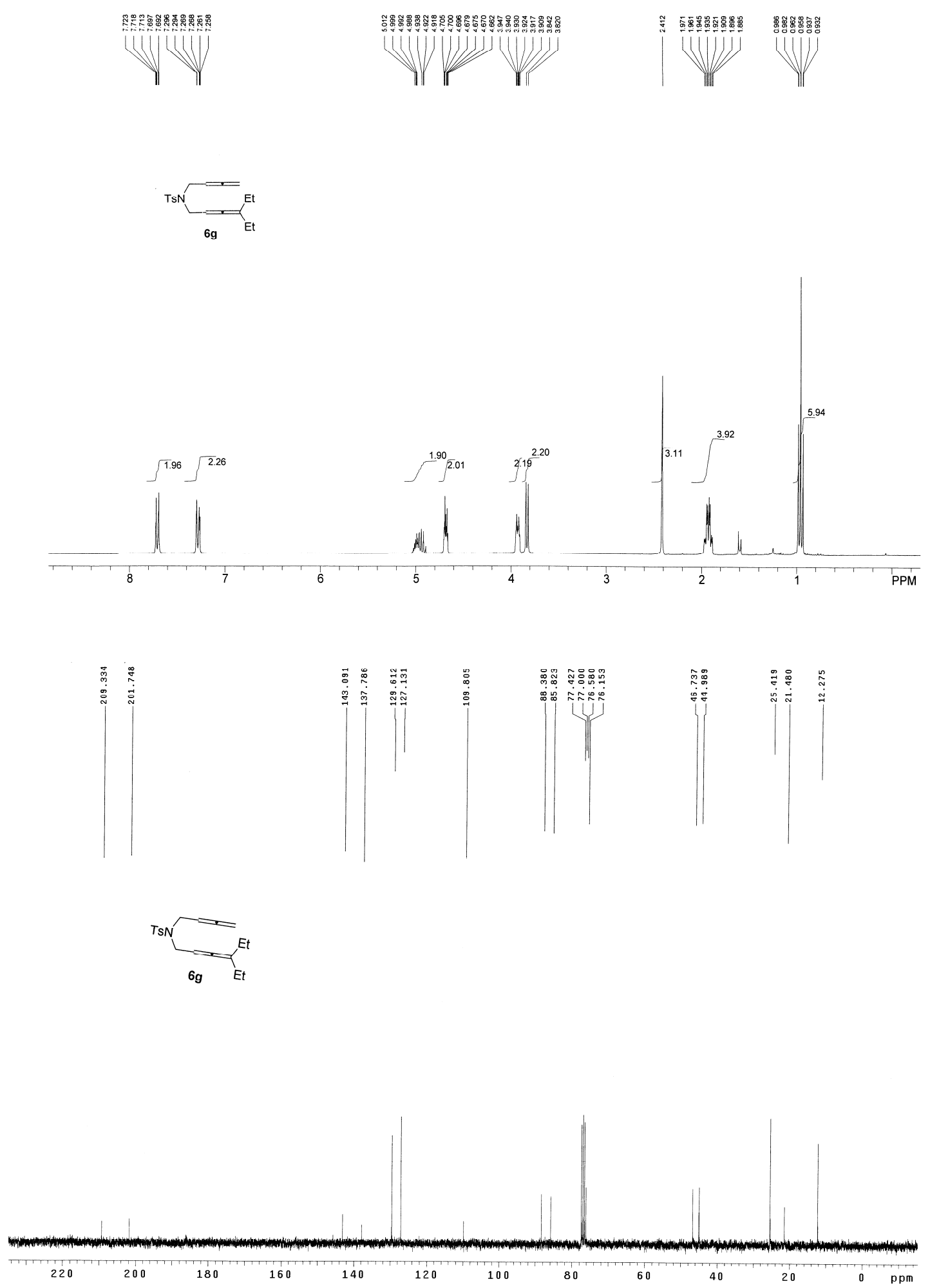


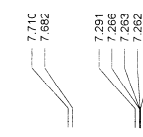

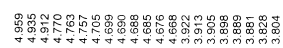

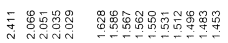

$\sqrt[T s N]{=}$

WWHe WH

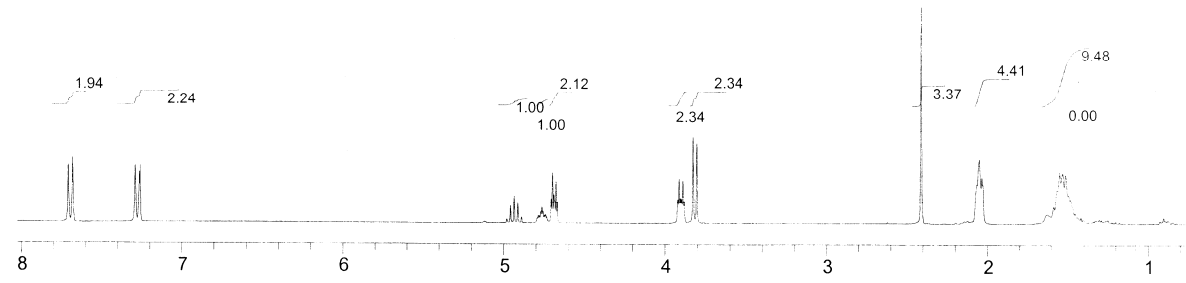

0 PPM
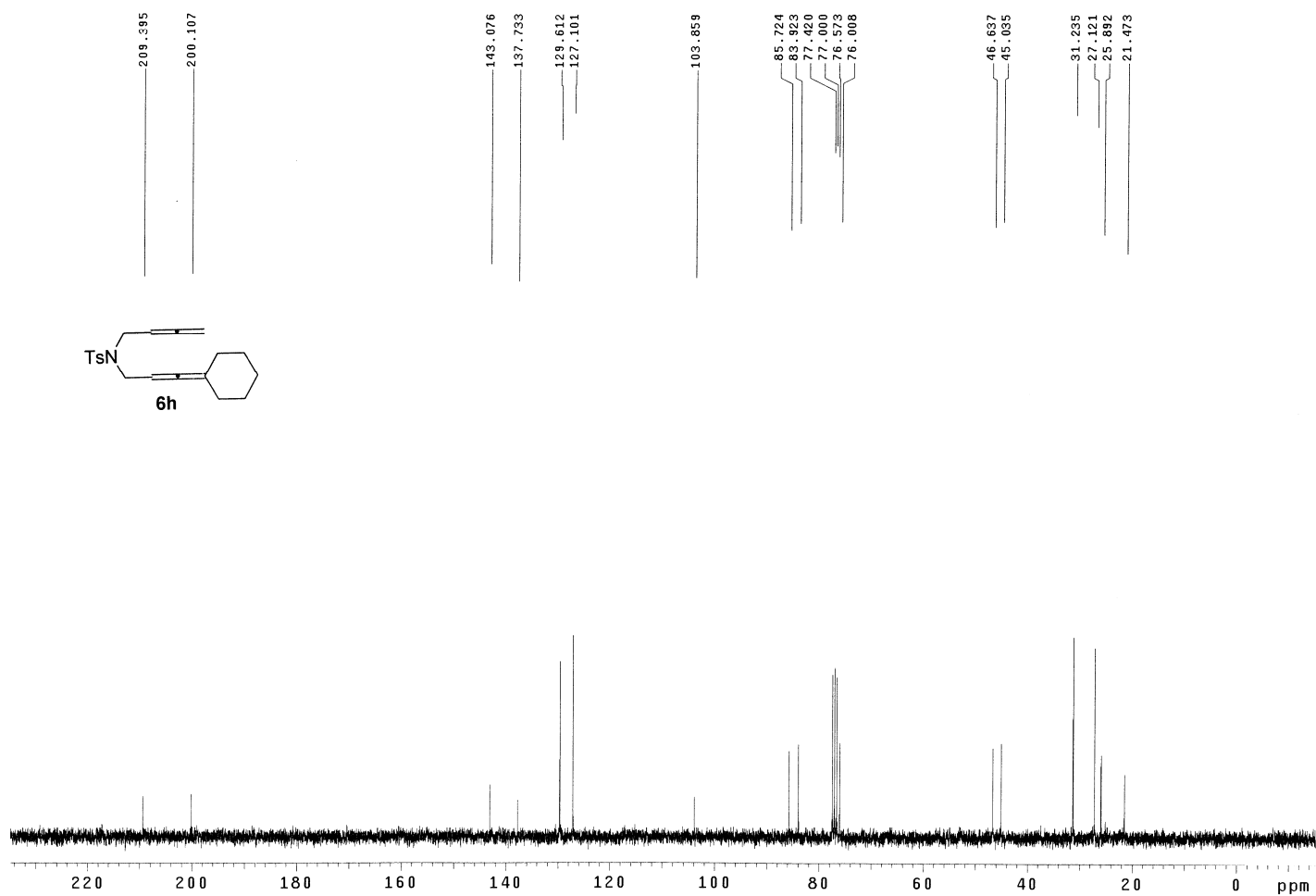

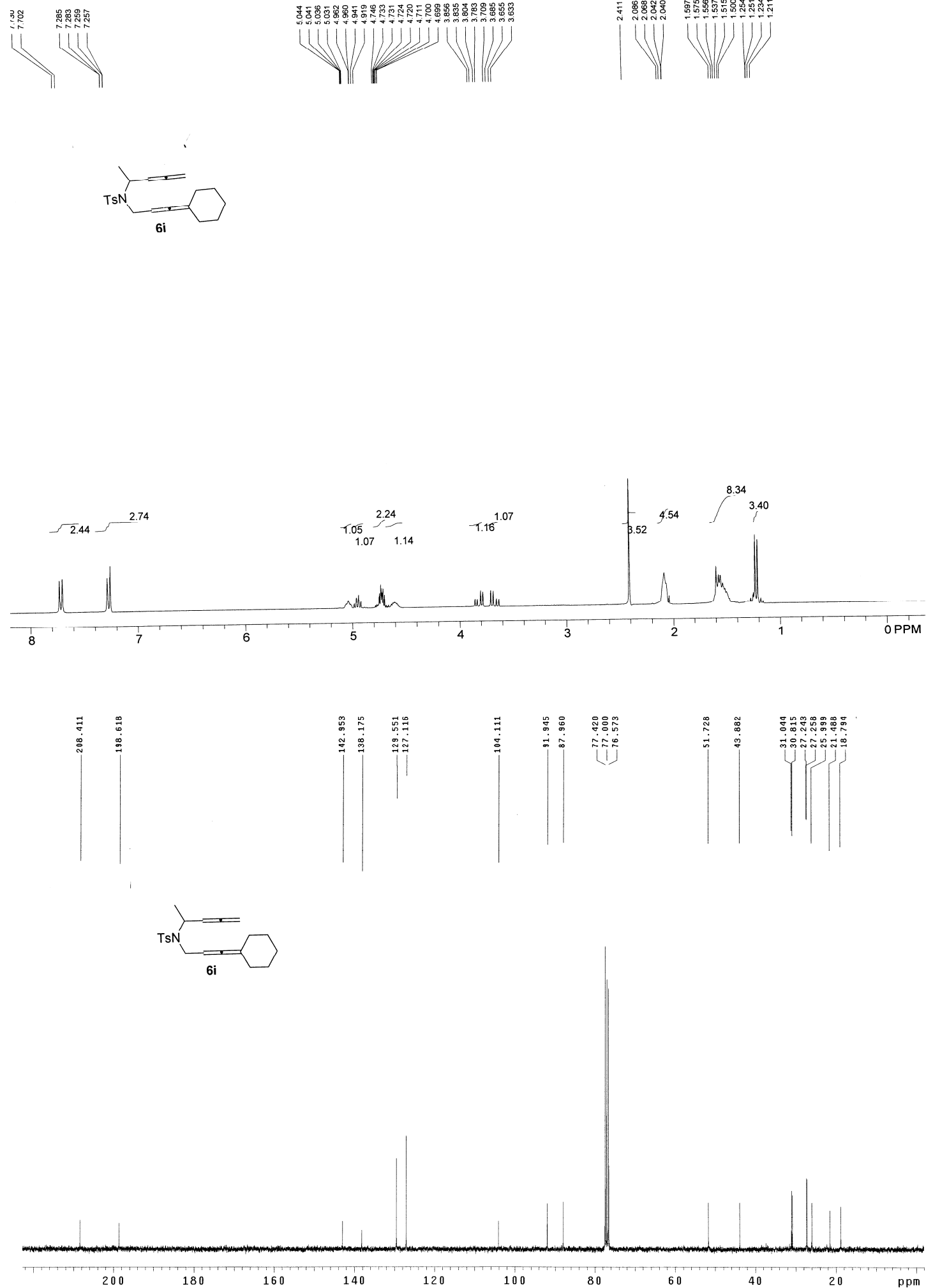


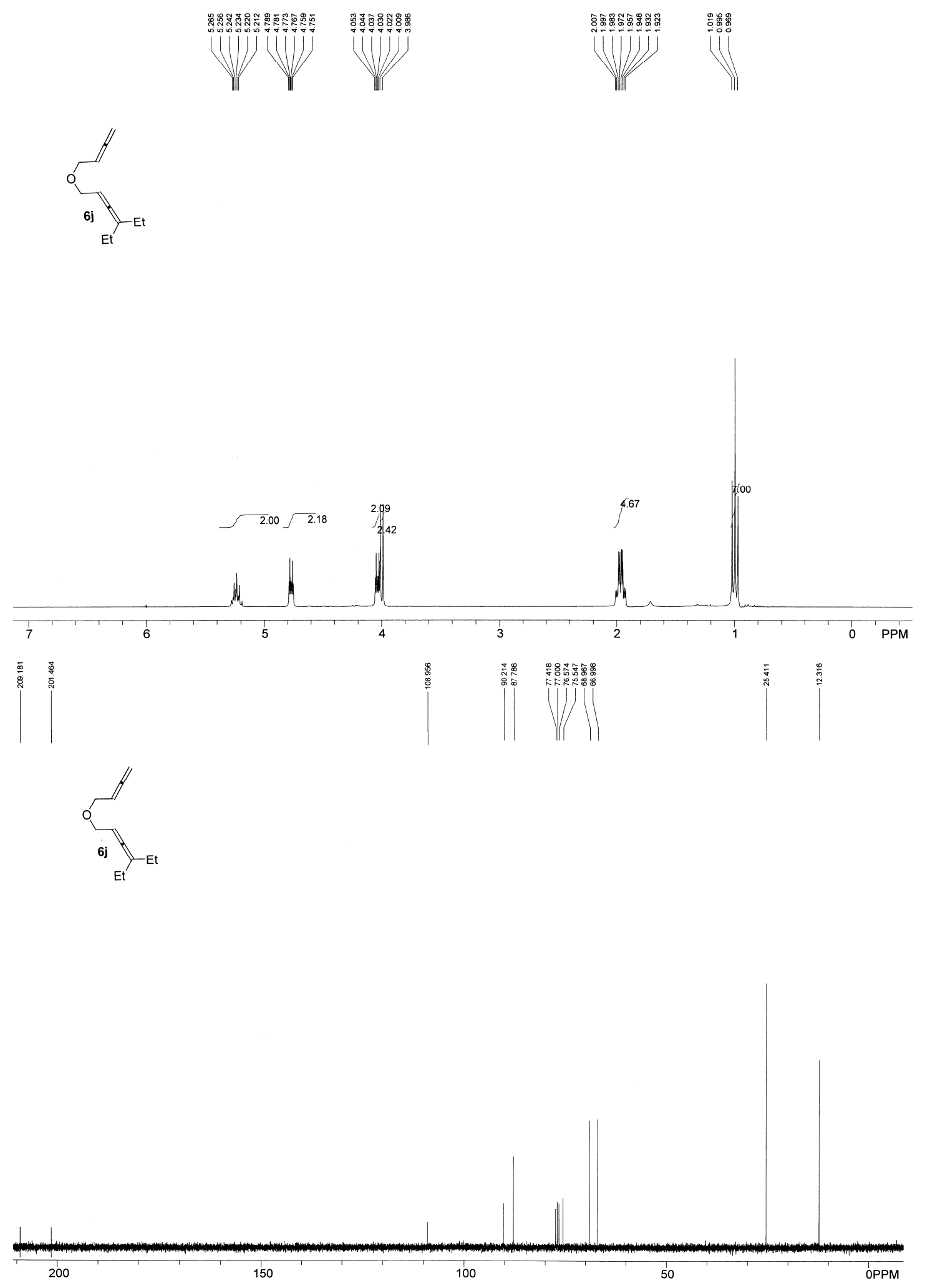




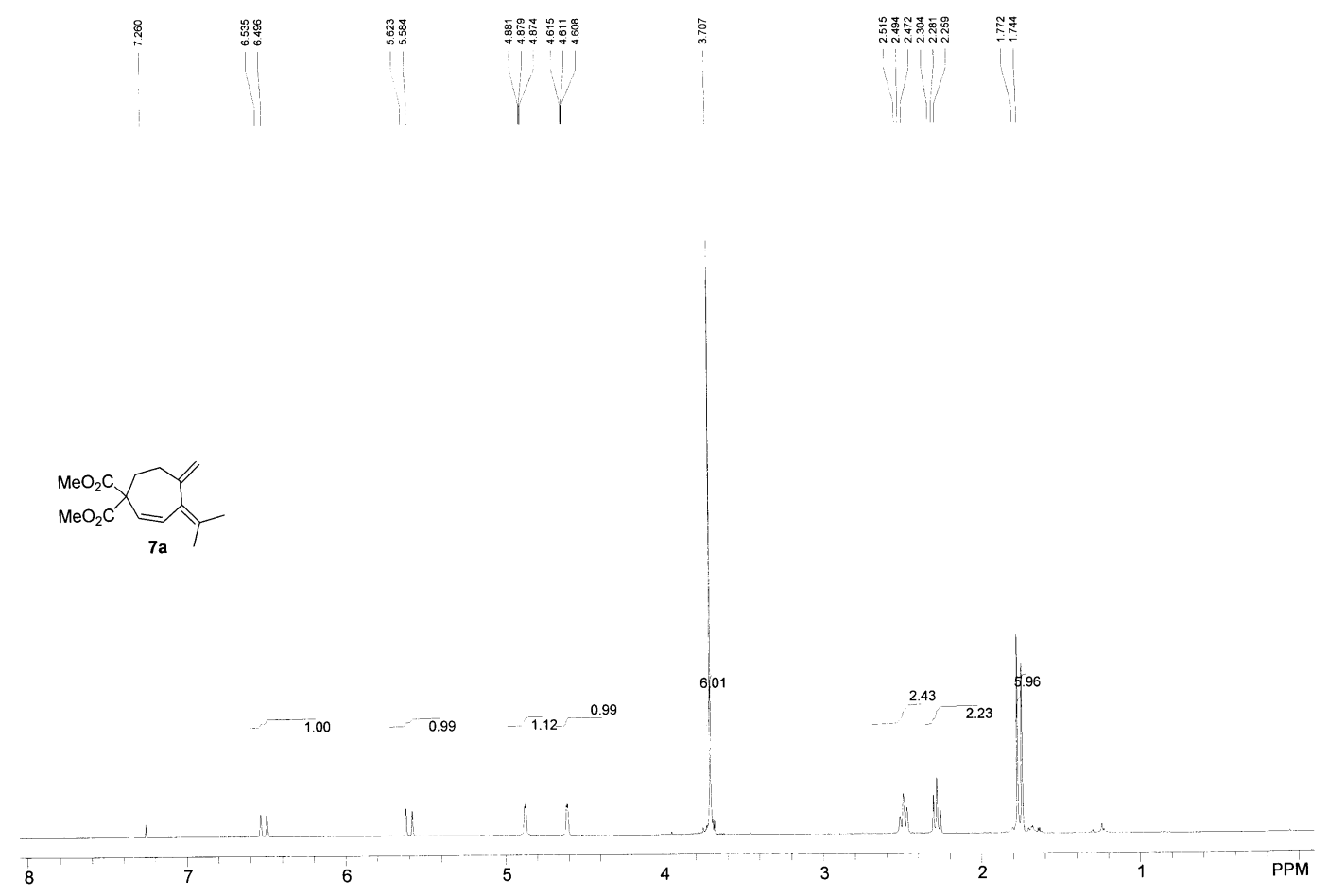

Archive directory: /export/home/masm/vnmrsys/data
Sample directory:

Pulse Sequence: s2pu

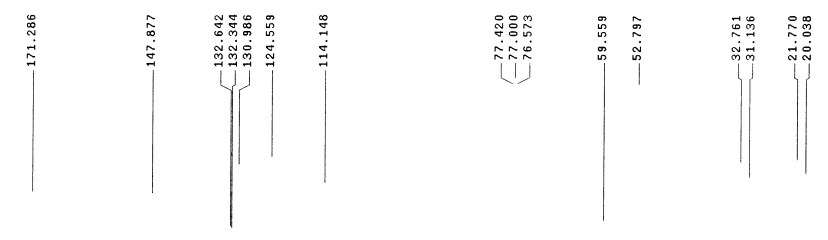

$\mathrm{MeO}_{2} \mathrm{C}$

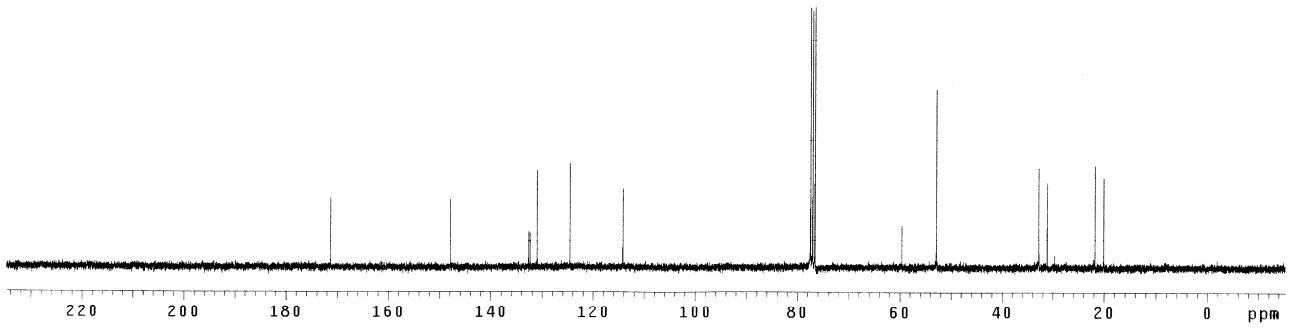



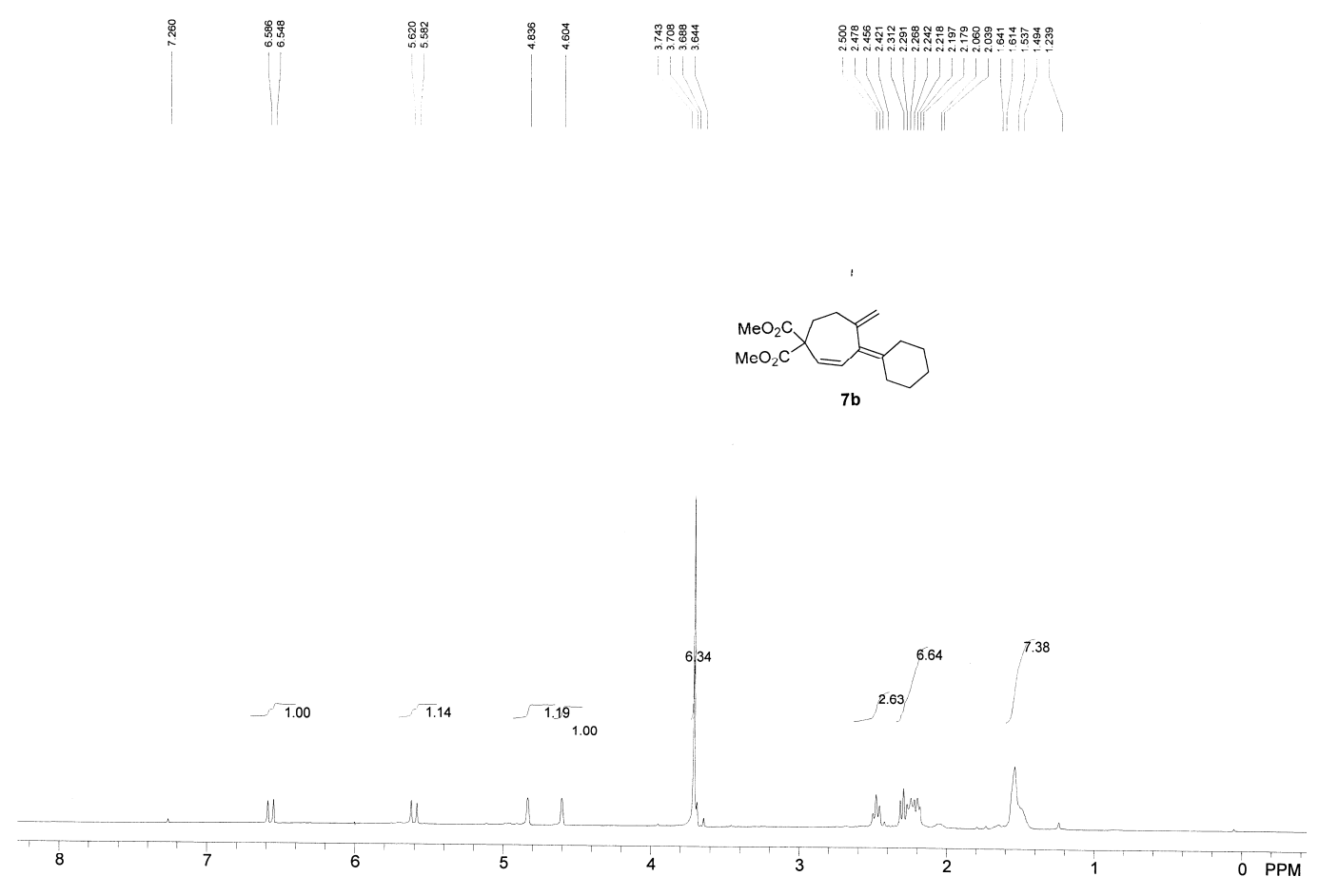

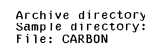

Pulse. Sequence: s2pur
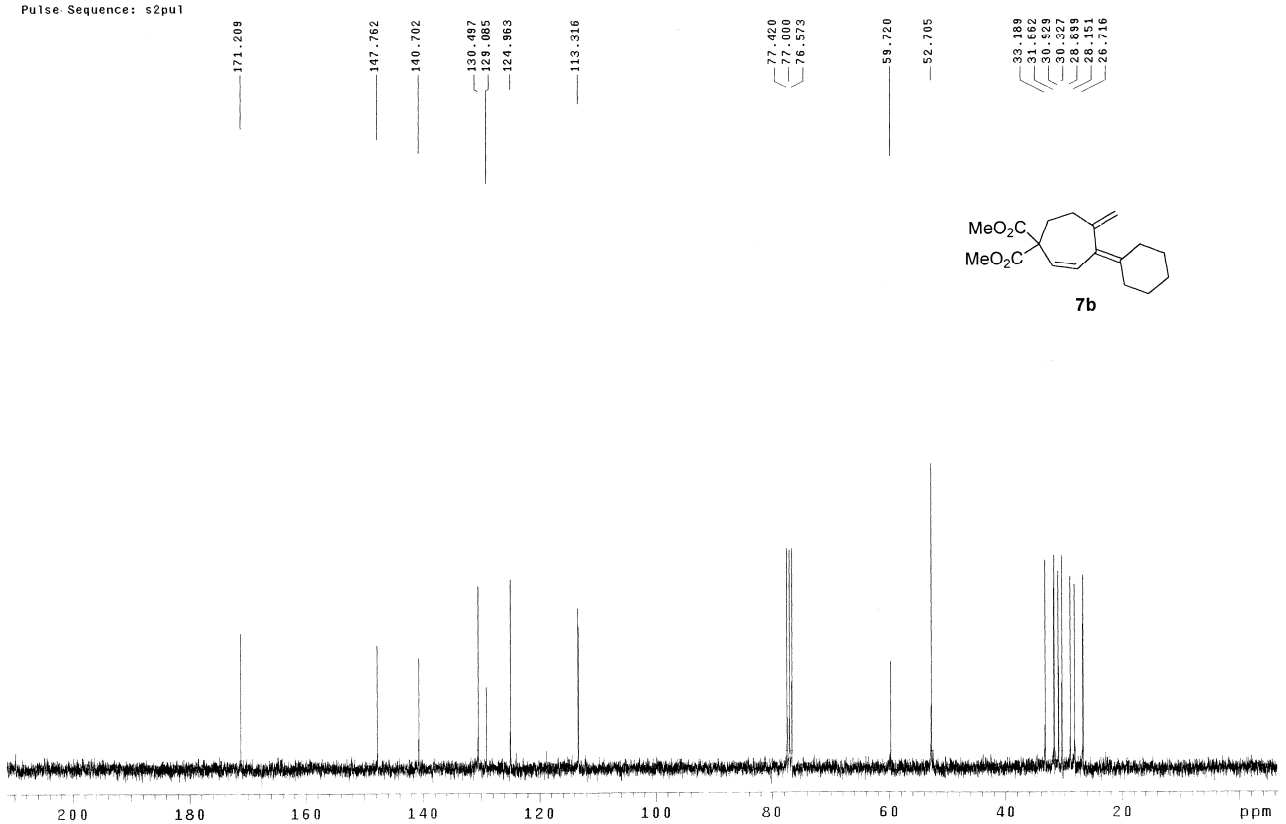

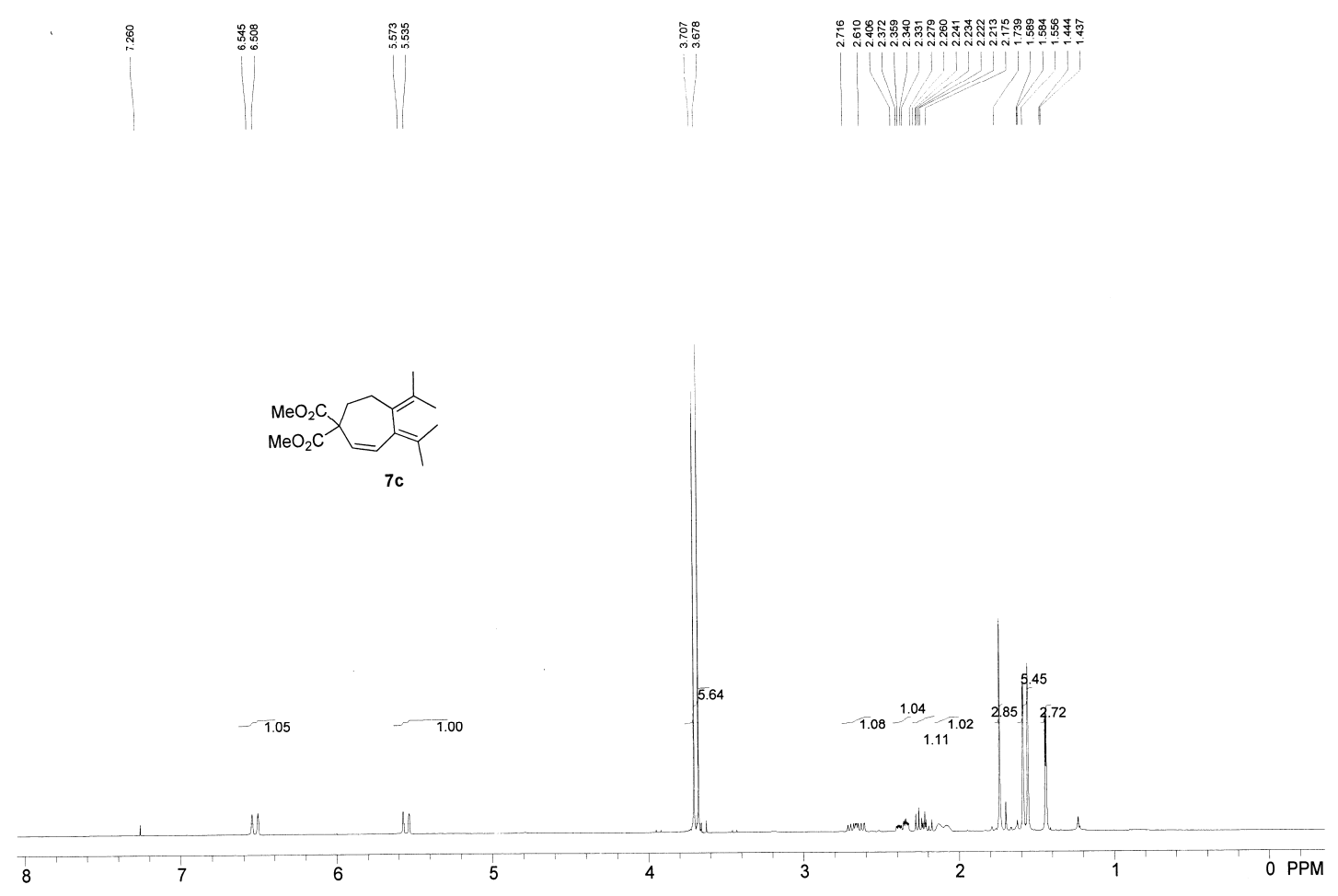

Archive directory: /oxport/homo/macm/vnmr sys/data
Sample directory:

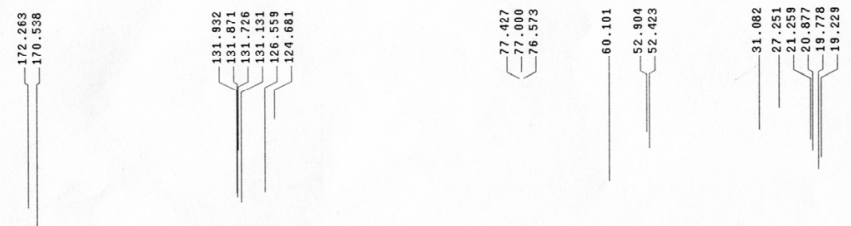

$\mathrm{MeO}_{2} \mathrm{C}$

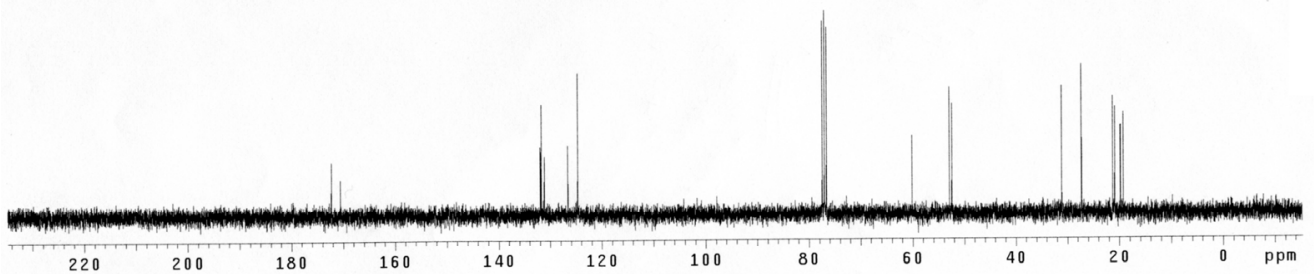



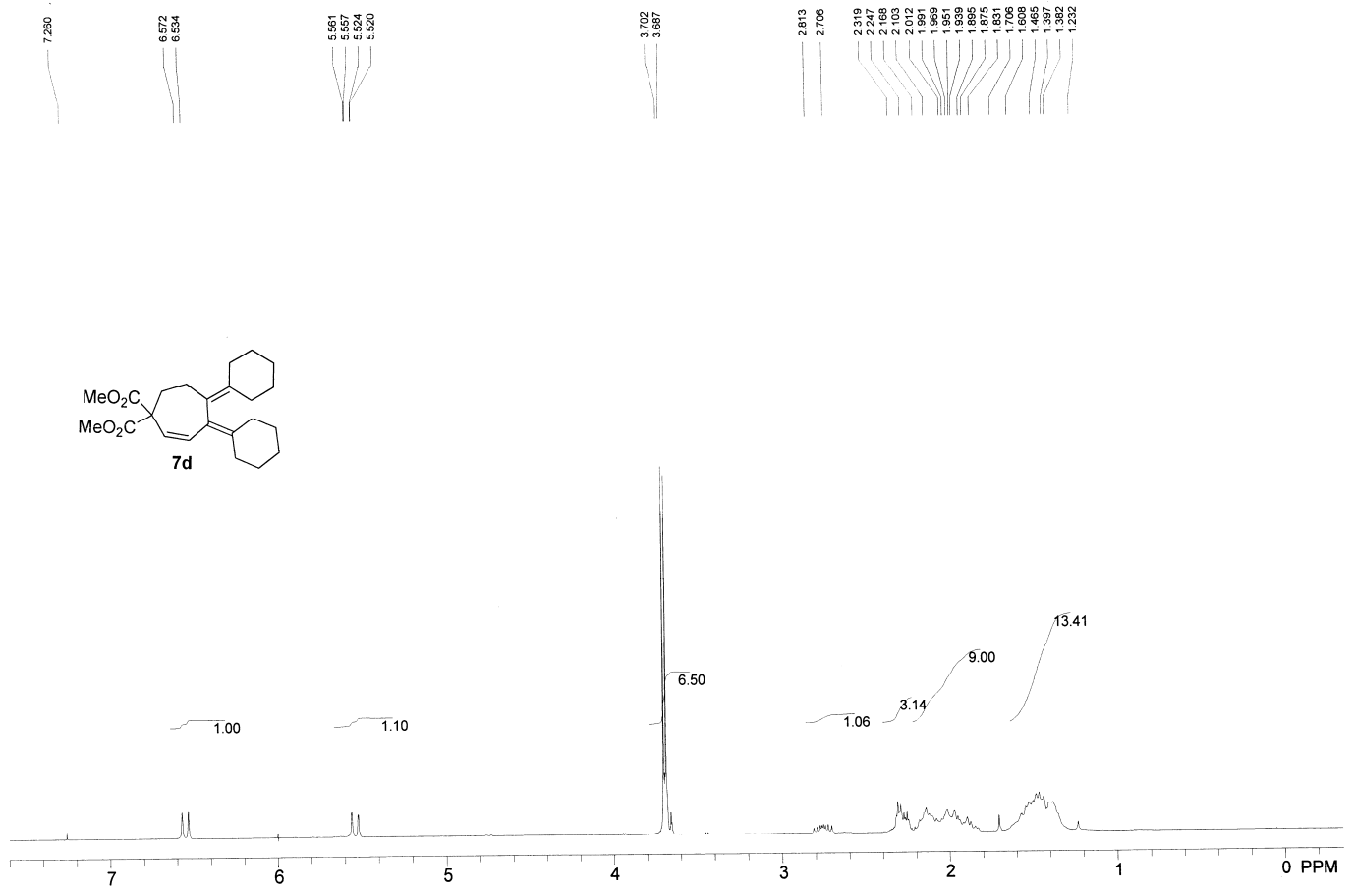

Archive directory: /export/home/masm/vnmrsys/data
Sample directory:

Pulse Sequence: s2pul
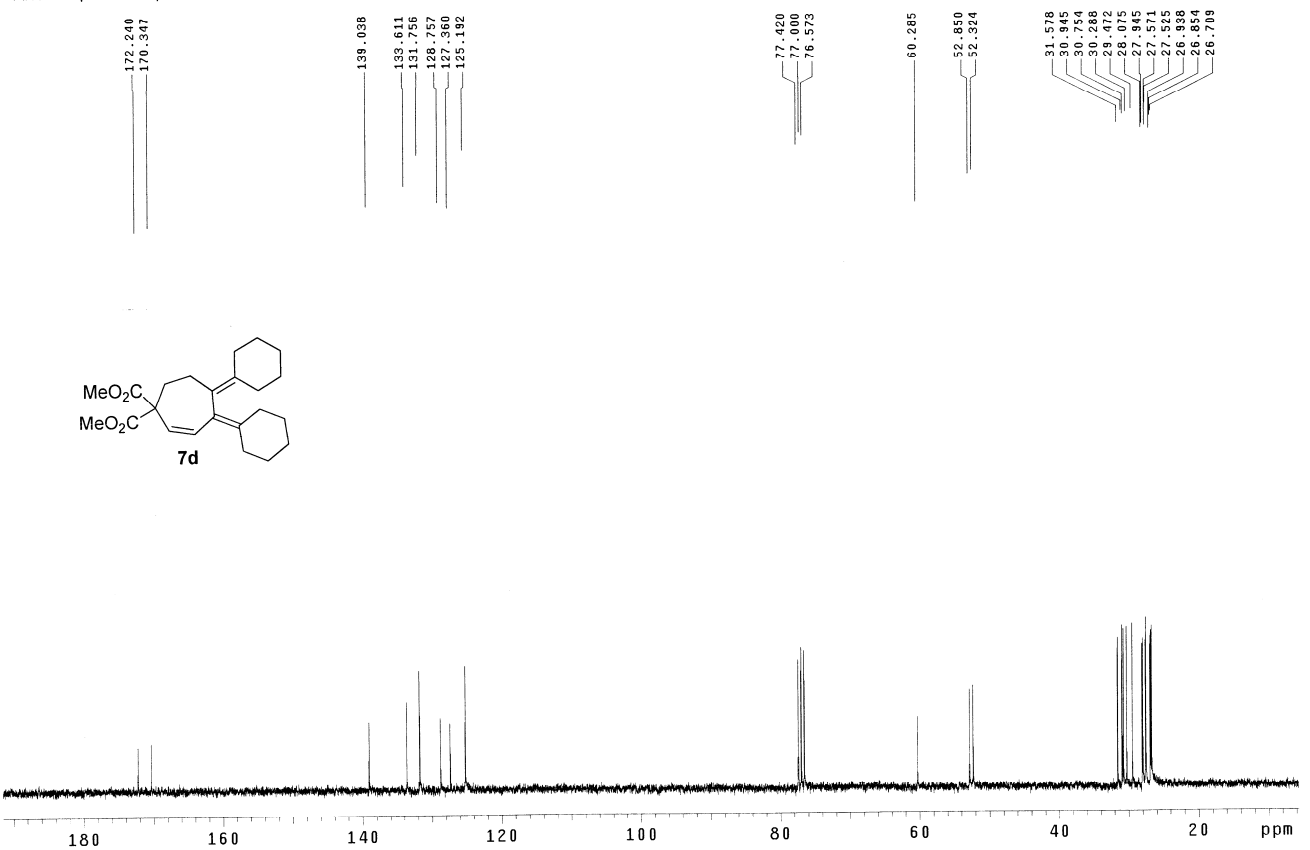


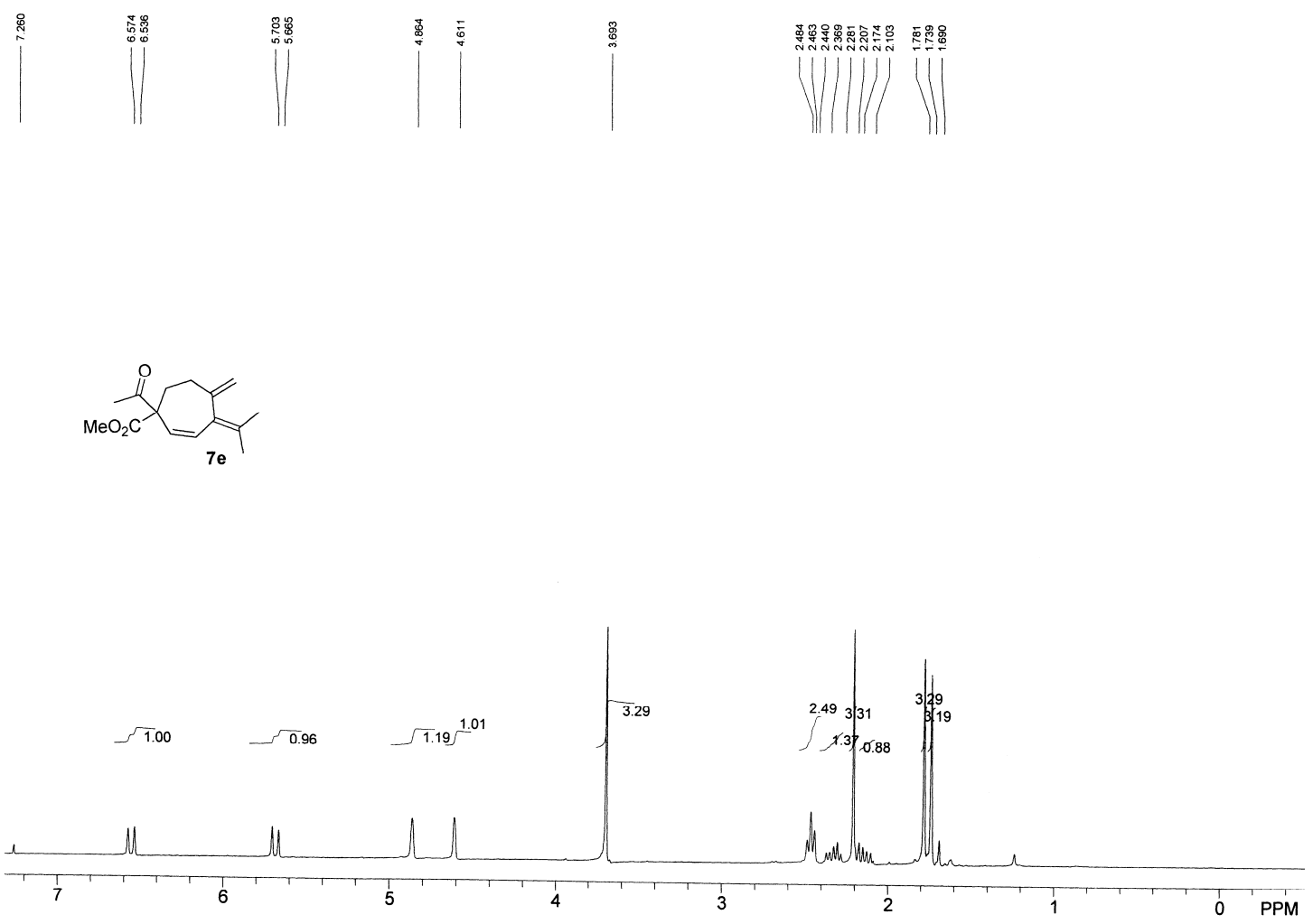

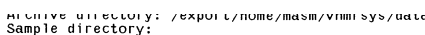
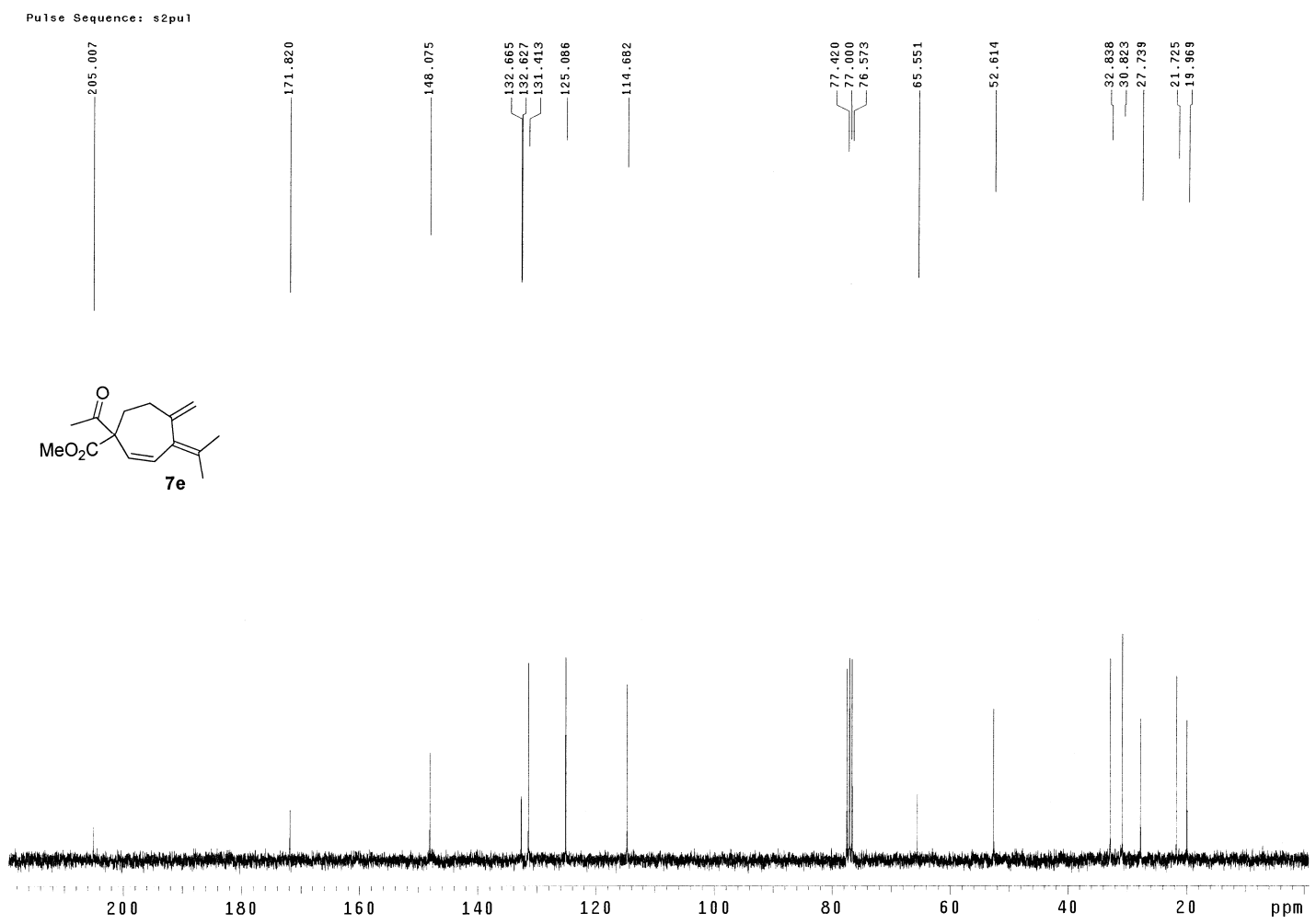


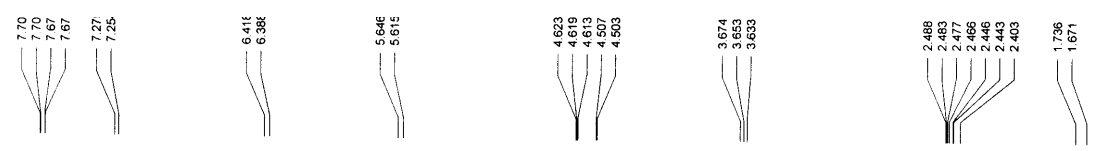
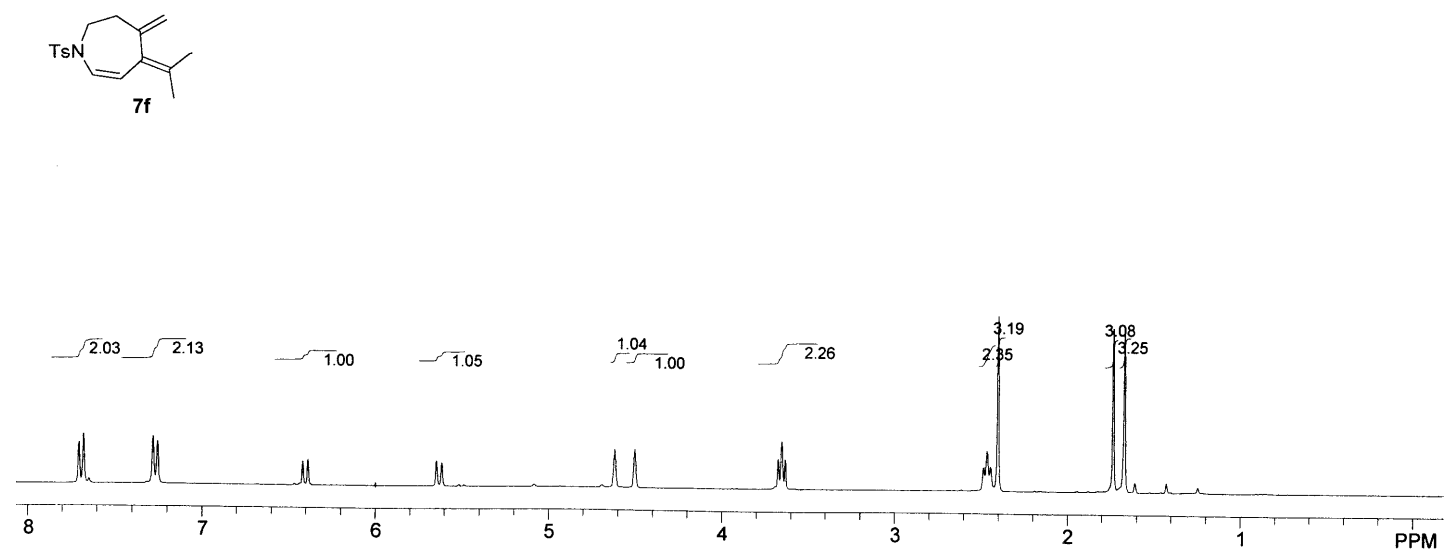

Archive directory: /export/home/masm/vnmrsys/data
Sample di rectory:

Pulse Sequence: s2pul

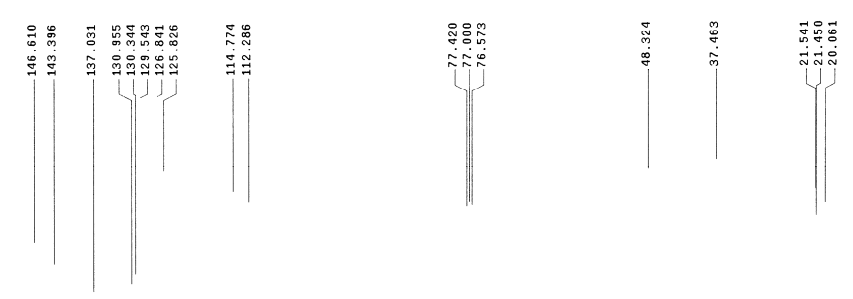

TsN

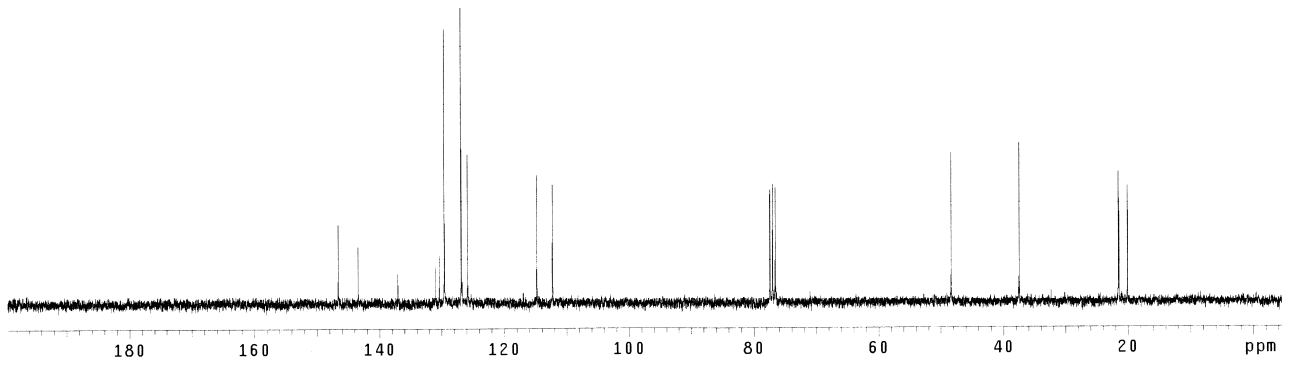




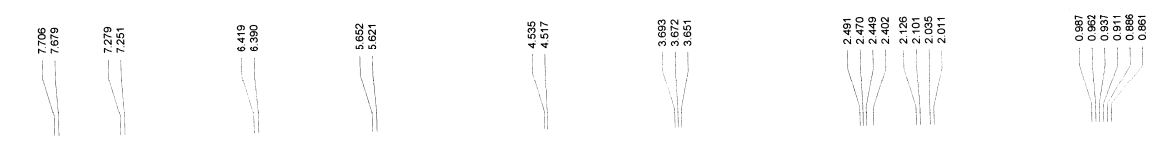

$$
\underbrace{E t}_{\mathbf{7 g}}
$$
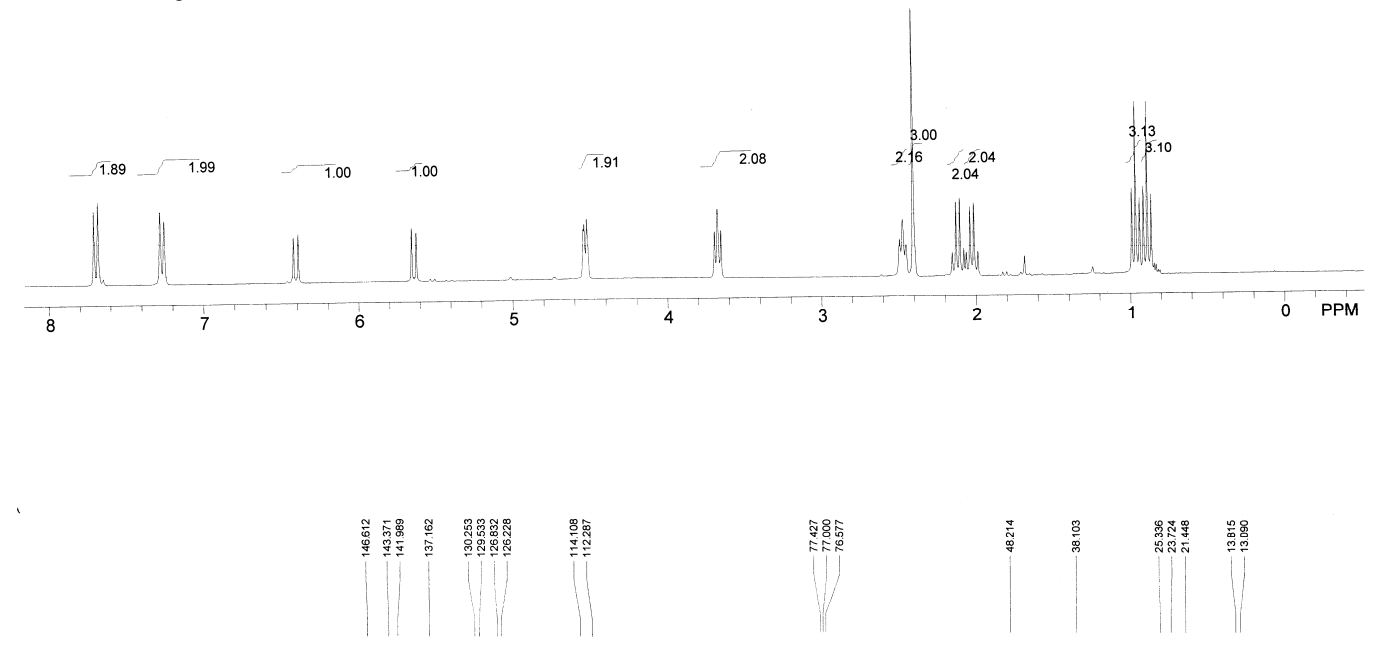

$\underbrace{\mathrm{Et}}_{\mathbf{7 g}}$

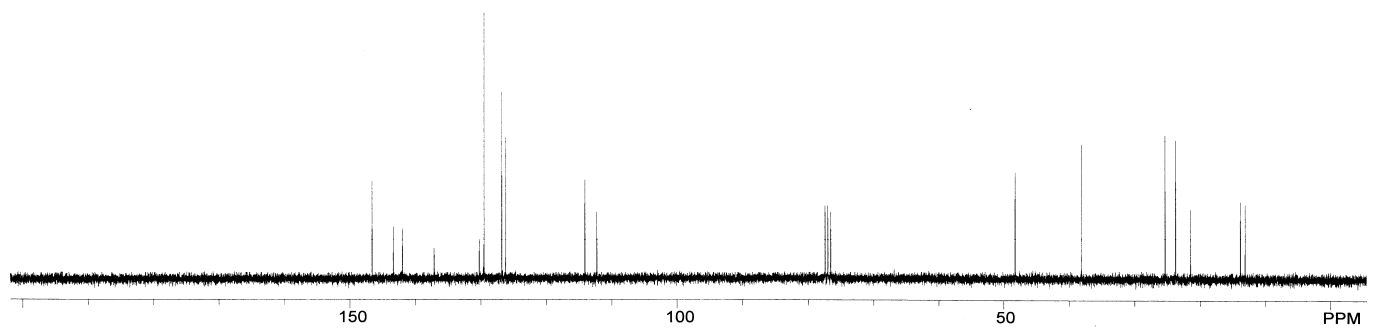




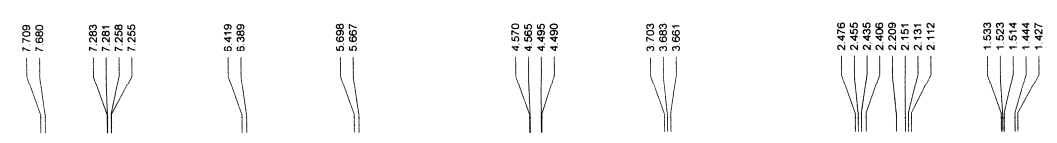

(s)
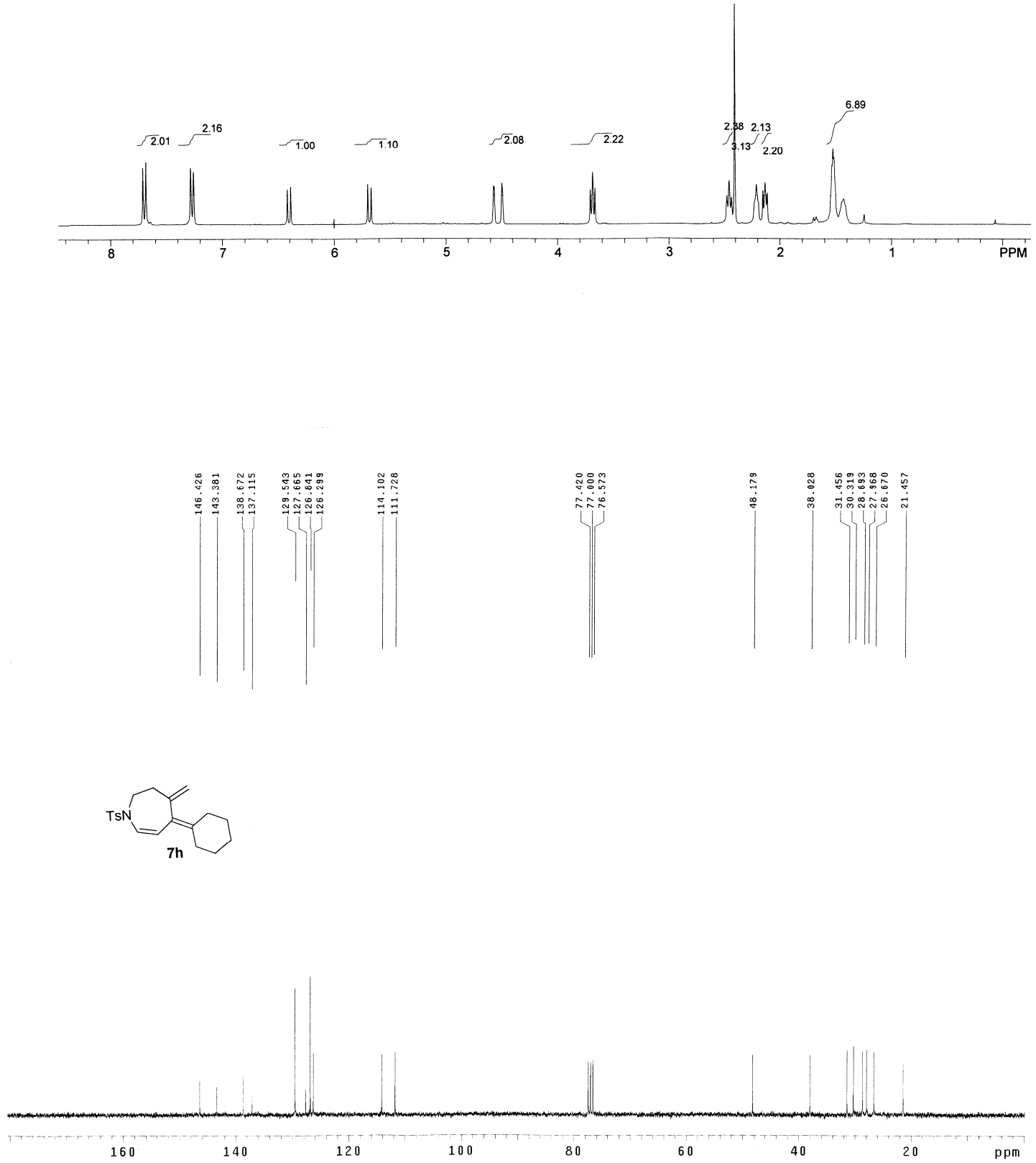


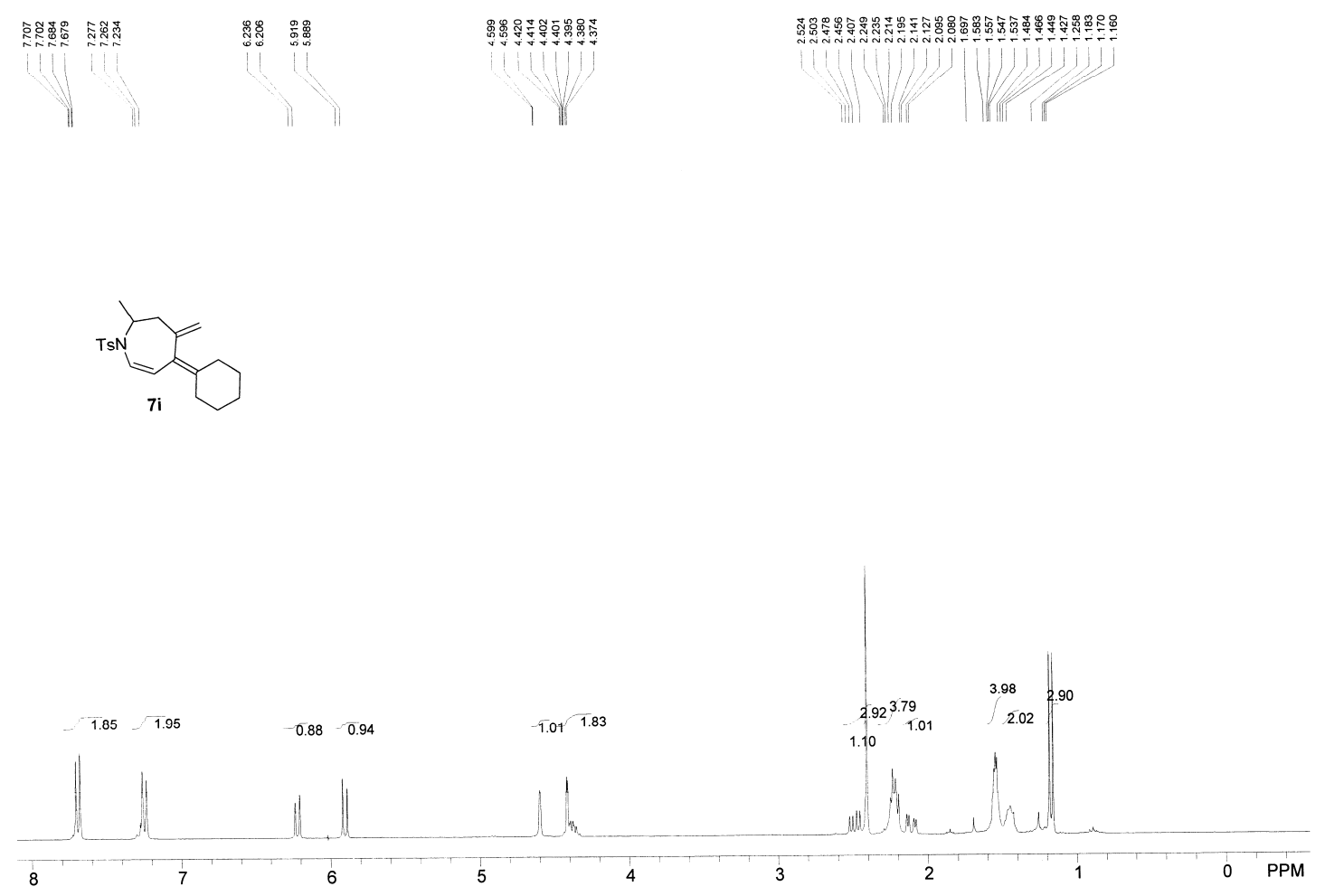

Archive directory: /export/home/masm/vnmrsys/data
Sample di rectory:

Pulse Sequence: s2pul
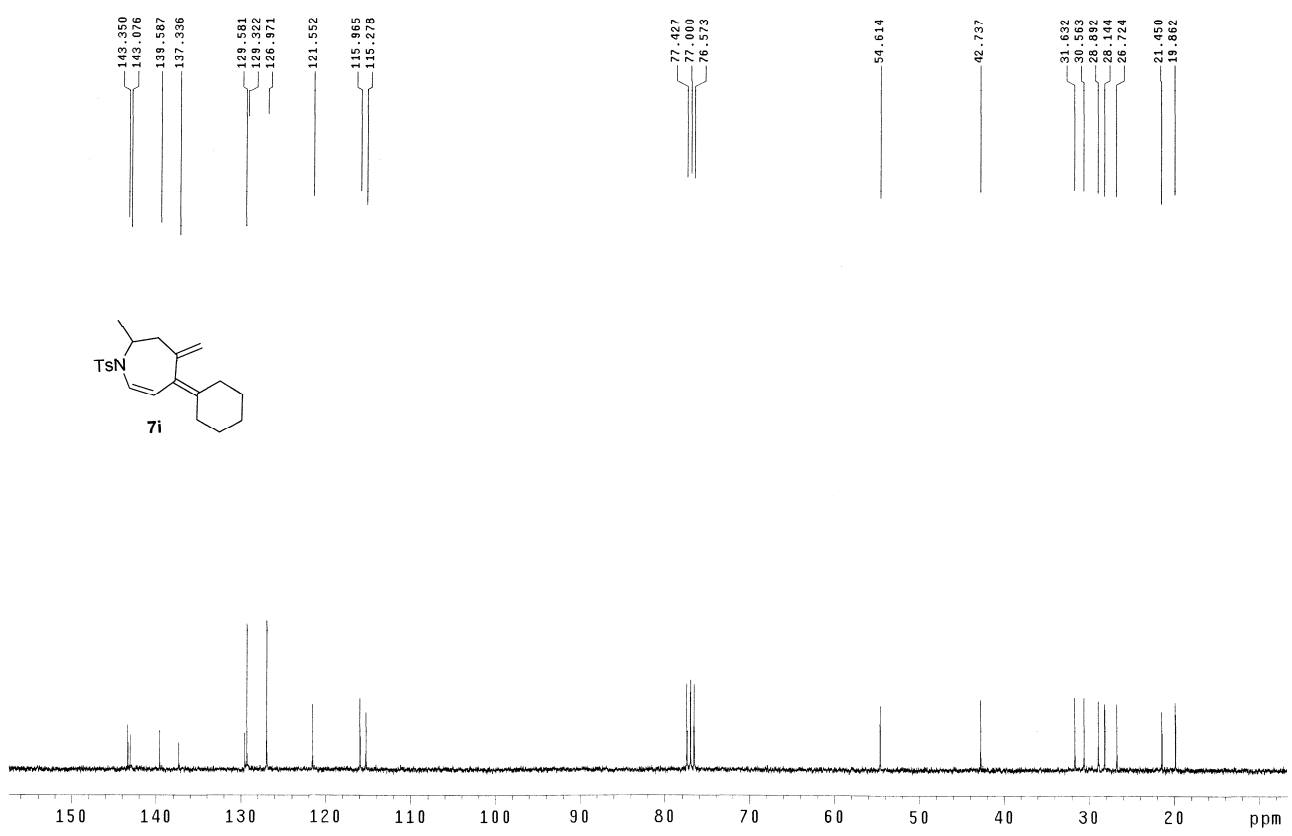

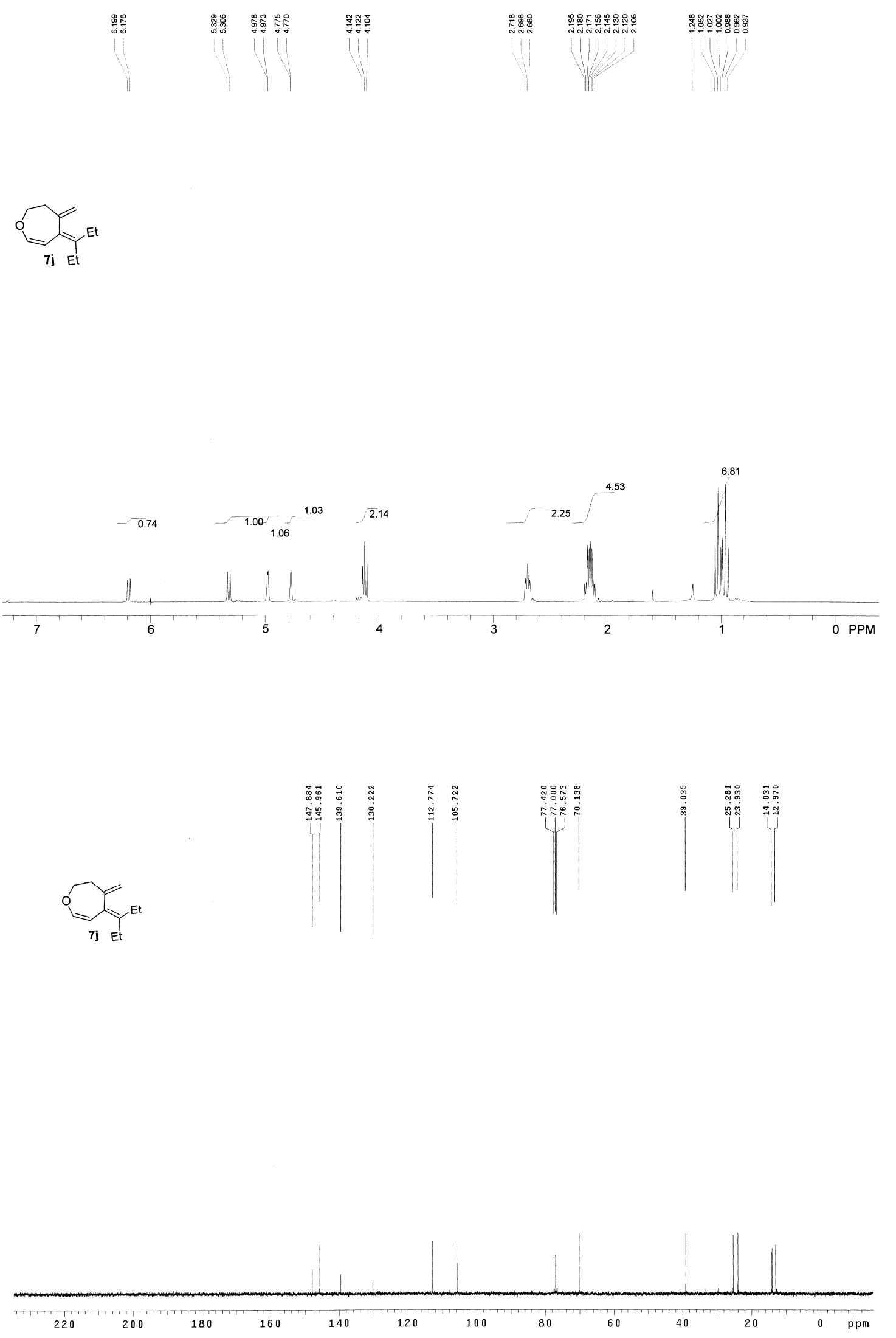


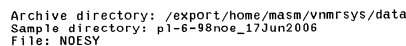
Pulse sequence: NOLSY Solvent: CDC13
Ambient temperature
Mercury-300BB "0MC300"
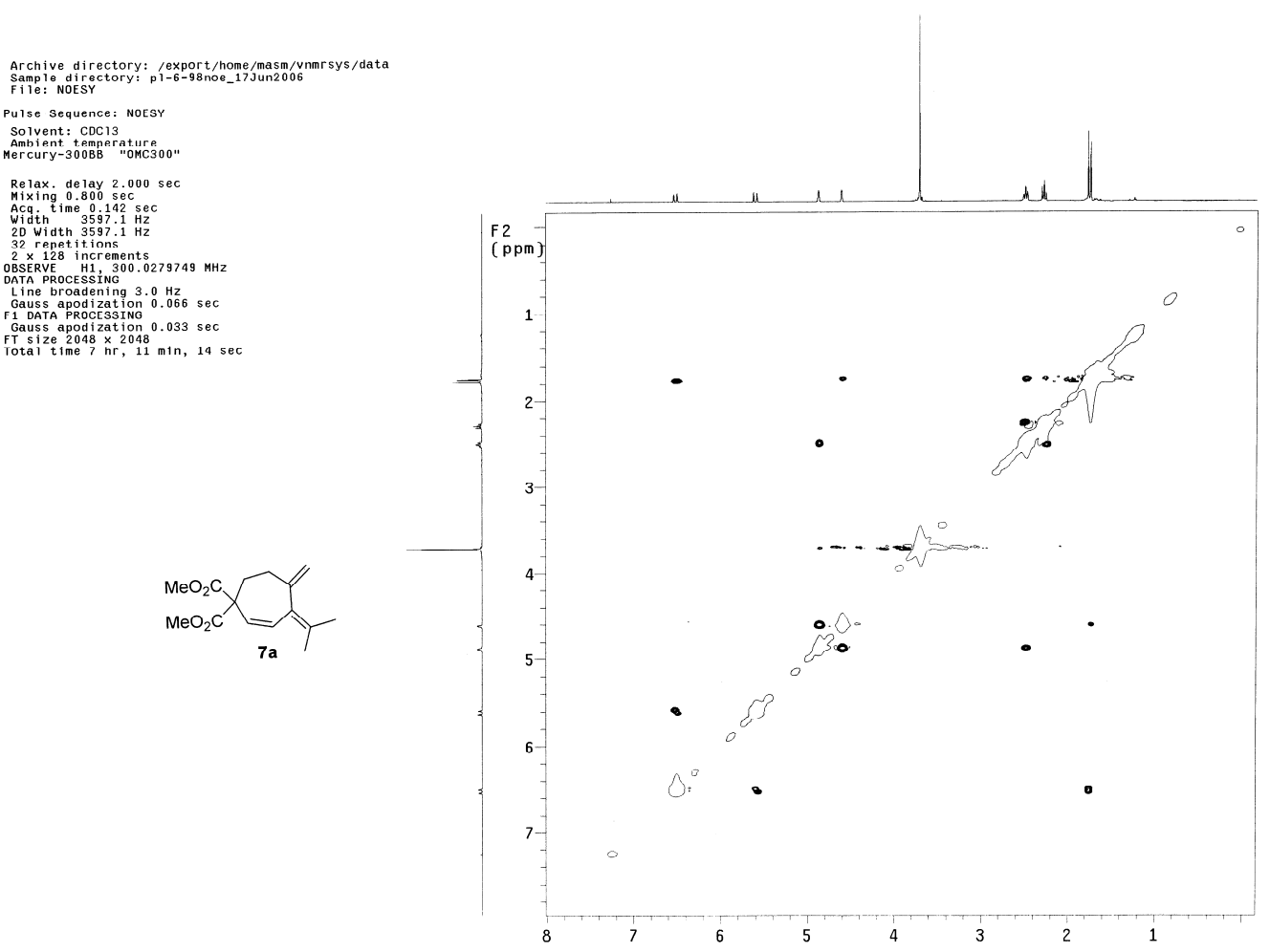

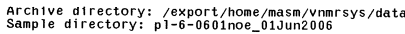

Pulse Sequence: NOESY
Solvent: CDC13

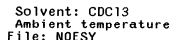

mercury-300BB "OMC300"

Relax. delay $2.000 \mathrm{sec}$
Mixing
Aix. 000 sec

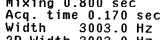

Width $3003.0 \mathrm{~Hz}$
20 width $3003.0 \mathrm{~Hz}$

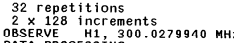

Gauss apodization $0.079 \mathrm{sec}$

Gauss apoodization $0.039 \mathrm{sec}$

FT size $2048 \times 2048.039 \mathrm{sec}$
Total time? $\mathrm{hr}, 15 \mathrm{~min}, 34 \mathrm{sec}$
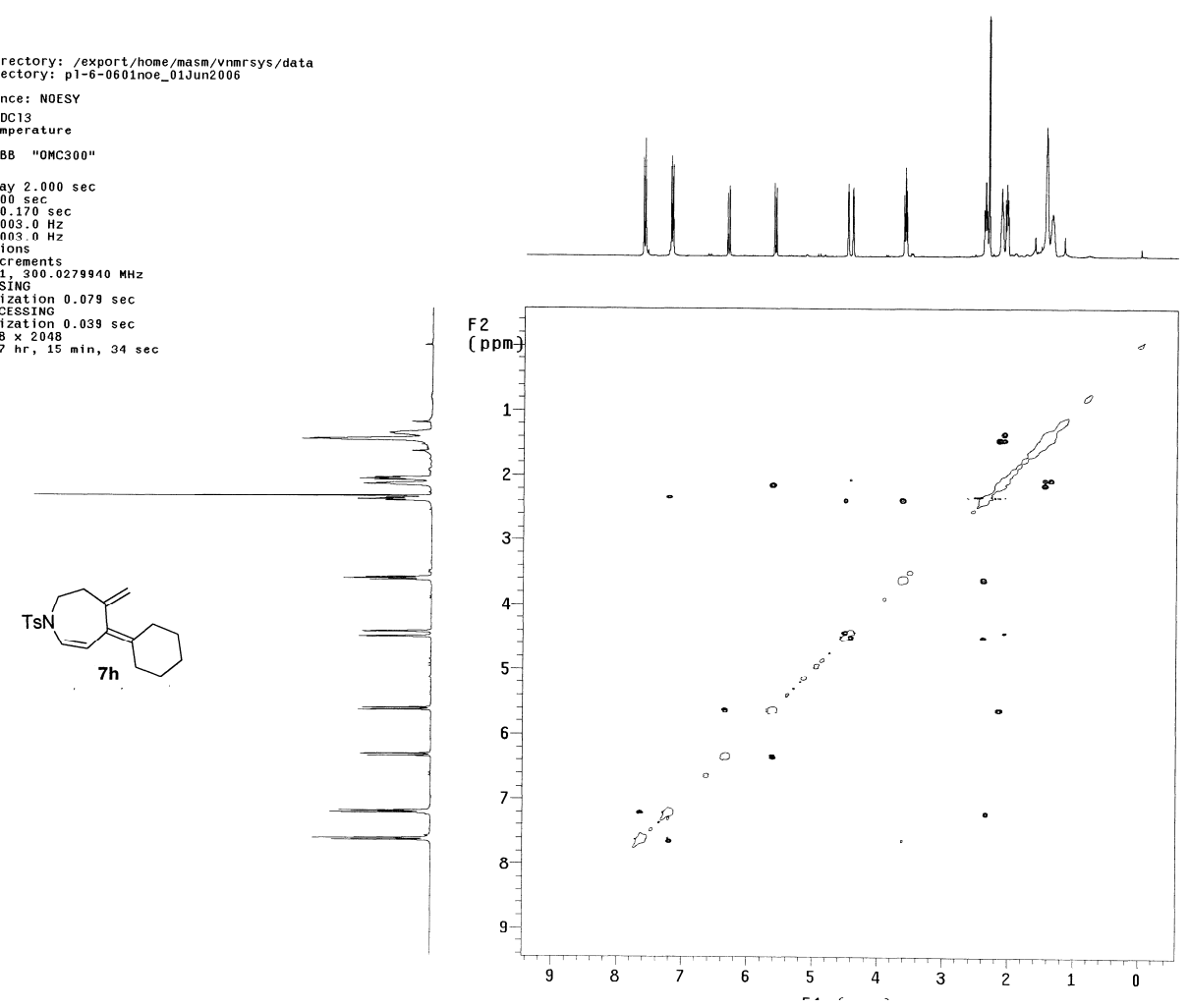
Archive directory: /export/home/masm/vnmrsys/data
Sample directory: p1-9-105-noe_14Apr2007
File: NOESY

Pulse Sequence: NOESY

Solvent: : CDC13
Ambient temperature
Mercury-300BB " 00 MC300

Relax. de lay $2.000 \mathrm{sec}$
Mixing $0.800 \mathrm{sec}$

Acq time 0.142 sec
Width $3597.1 \mathrm{~Hz}$

32 repetitions $35 \mathrm{~Hz}$

$2 \times 128$ increments
OBSERVE H1, $300.0279749 \mathrm{MHZ}$

TA PROCESSING

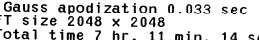

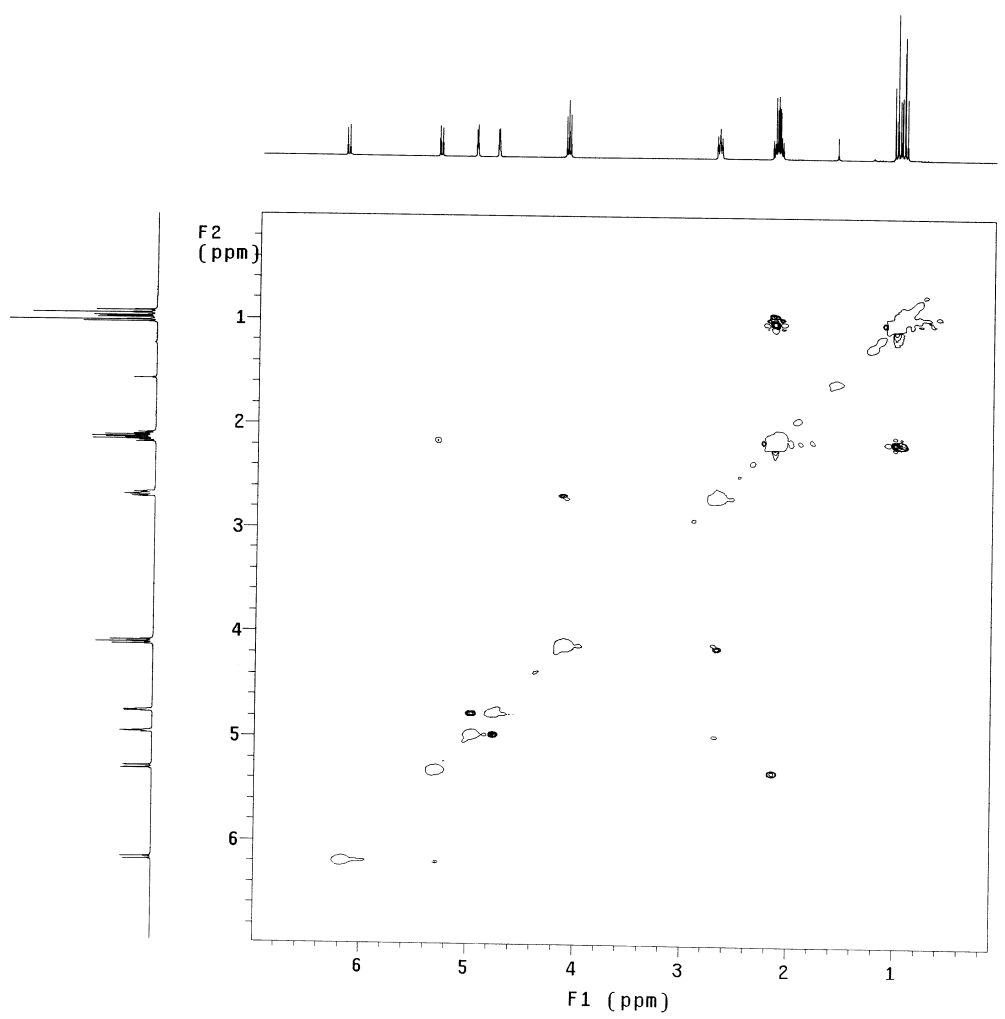

